\title{
Familias de polinômios estáveis: teoremas de Routh-Hurwitz e Kharitonov
}

\author{
Seong Ho Lee
}

\author{
DisSERTAÇÃO APRESENTADA \\ $\mathrm{AO}$ \\ Instituto de Matemática e EstatísticA \\ DA \\ Universidade de SÃo Paulo \\ PARA \\ OBTENÇÃO DO TÍTULO \\ $\mathrm{DE}$ \\ Mestre em CiÊnCIAS \\ Programa: Matemática Aplicada \\ Orientador: Jorge Manuel Sotomayor Tello
}

Durante o desenvolvimento deste trabalho o autor recebeu auxílio financeiro da CAPES

São Paulo, 26 de agosto de 2008 


\section{Familias de polinômios estáveis: teorema de Routh-Hurwitz e Kharitonov}

Este exemplar corresponde à redação final da dissertação devidamente corrigida e defendida por Seog Ho Lee e aprovada pela Comissão Julgadora.

Banca Examinadora:

- Prof. Dr. Jorge Manuel Sotomayor Tello (orientador) - IME-USP.

- Prof. Dr. Manuel Valentin de Pera Garcia - IME-USP.

- Prof. Dr. Ronaldo Alves Garcia - UFG. 
A Kyung Hyun Lee e Kyung Hye Cha Lee. 


\section{Agradecimentos}

Em primeiro lugar, sou grato ao Prof. Jorge Sotomayor Tello pela orientação, incentivo e apoio na elaboração deste trabalho. Aprendi e continuo aprendendo como um pesquisador deveria trabalhar.

Sou grato ao Prof. Manuel Valentim de Pera Garcia por ter me transmitido o seu entusiasmo pelo estudo da matemática.

Aos meus pais Kyung Hyun e Kyung Hye que me deram todo apoio. Aos meus amigos que estavam presentes quando precisei deles. Quero agradecer também aos colegas de turma 9 do Curso de Ciências Moleculares.

Por fim, agradeço aos todos amigos e professores que ajudaram na revisão deste dissertação. 


\section{Resumo}

O objetivo deste trabalho é caracterizar os polinômios cujas raízes têm todas parte real negativa, chamados de polinômios estáveis ou de Hurwitz. Para este fim, apresentaremos e provaremos o critério de Routh-Hurwitz. Também estenderemos este resultado para obter uma caracterização da estabilidade para uma família de polinômios com seus coeficientes variando independentemente num intervalo limitado. Aplicaremos os resultados para obter um critério de estabilidade robusta para um sistema de equações diferenciais que descreve um sistema mecânico.

Palavras-chave: Routh, Hurwitz, Kharitonov. 


\section{Abstract}

The objective of this work is to determine when all of zeros of a given polynomial have negative real parts, called stable or Hurwitz polynomials. We will present and prove the Routh-Hurwitz criterion. Furthermore we will extend the result for classes of polynomials defined by letting their coefficients vary independently in an arbitrary finite interval. Then we will apply them to derive a robust stability condition for a mechanical system.

Keywords: Routh, Hurwitz, Kharitonov. 


\section{Sumário}

Lista de Figuras $\quad$ xi

\begin{tabular}{lll}
\hline & Introdução & 1
\end{tabular}

2 Preliminares $\quad 5$

2.1 Estabilidade de Liapunov $\ldots \ldots \ldots \ldots \ldots \ldots \ldots$

2.2 Equações lineares com coeficientes constantes $\ldots \ldots \ldots \ldots$

3 Critério de estabilidade $\quad 9$

3.1 Propriedades dos polinômios de Hurwitz $\ldots \ldots \ldots \ldots$. . . . . . . . . . . . . . 9

3.1 .1 Condição necessária $\ldots \ldots \ldots \ldots \ldots \ldots \ldots$

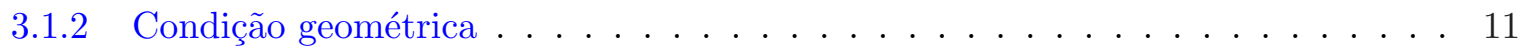

3.2 Critérios de Routh e Hurwitz . . . . . . . . . . . . . . . . . . . . . . . 20

3.2 .1 Construção de esquema de Routh $\ldots$. . . . . . . . . . . . . . . . . . . 22

3.2.2 Construção de uma seqüência de polinômios a partir de esquema de Hurwitz . 26

$3.2 .3 \quad$ Teorema de Routh e de Hurwitz $\ldots \ldots \ldots$. . . . . . . . . . . 37

4 Teorema de Kharitonov 43

4.1 Teorema de Kharitonov $\ldots \ldots \ldots$. . . . . . . . . . . . . . . . 43

4.1 .1 Teorema de Kharitonov . . . . . . . . . . . . . . . . . . . . . . . 43 
4.1 .2 Uma simplificação para os polinômios de grau menor que 6 . . . . . . . . . 48

4.2 Aplicação do teorema de Kharitonov ～. . . . . . . . . . . . . . . . . . . . . . 51

\begin{tabular}{|lrl}
5 & Conclusões & 63
\end{tabular}

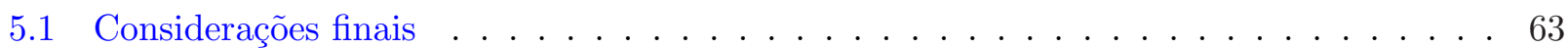

5.2 Sugestões para estudo adicional $\ldots \ldots \ldots \ldots \ldots$. . . . . . . . . . . 66

$\begin{array}{ll}\text { Referências Bibliográficas } & 69\end{array}$

\begin{tabular}{ll}
\hline Índice Remissivo & 71
\end{tabular} 


\section{Lista de Figuras}

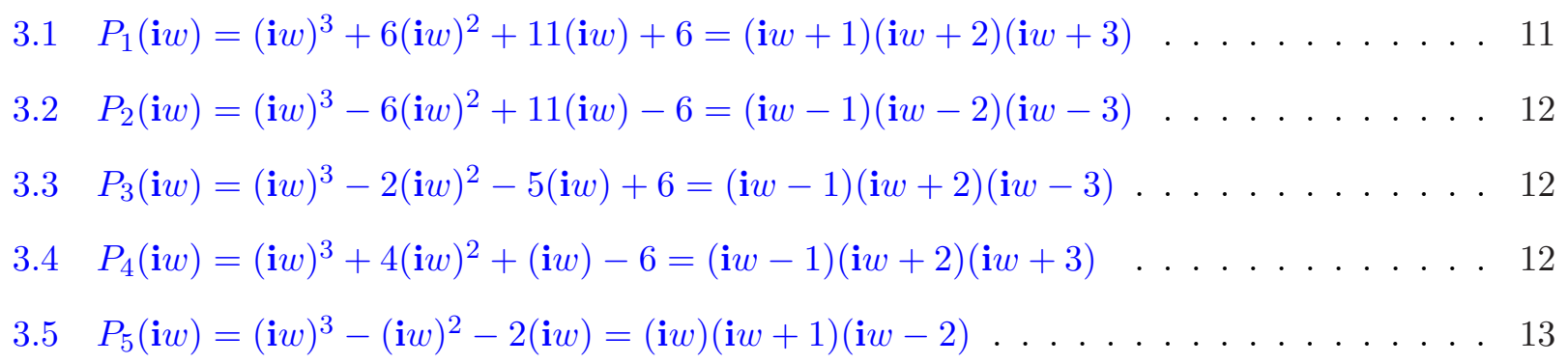

3.6 Tangente para as raízes com parte real negativa . . . . . . . . . . . . . . . . . 14

$3.7 \quad$ Montonicidade estritamente crescente de $\arg (P(\mathbf{i} w)) \quad \ldots \ldots \ldots \ldots$

3.8 Tangente para as raízes com parte real positiva $\ldots \ldots \ldots \ldots$

3.9 diagrama de Leonhard para $\mathrm{P}(\mathrm{z}) \ldots \ldots \ldots \ldots \ldots \ldots \ldots \ldots \ldots$

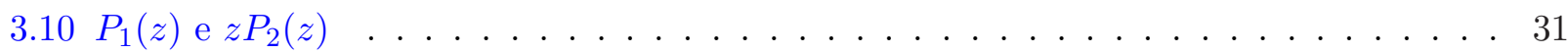

3.11 A região definida pelas desigualdades (3.73) no plano complexo . . . . . . . . . . . . . 41

$4.1 \quad$ A imagem do retângulo $M(w) \ldots \ldots \ldots \ldots \ldots \ldots$

$4.2 \quad$ A contradição quando $0 \in M(w) \ldots \ldots \ldots \ldots \ldots$

4.3 Polinômios de grau $4 \ldots \ldots \ldots \ldots \ldots \ldots \ldots$

4.4 manipulador . . . . . . . . . . . . . . . . . . . . . 56 


\section{Capítulo 1}

\section{Introdução}

O objetivo principal deste trabalho é caracterizar os polinômios cujas raízes possuem todas partes reais negativas, denominados polinômios estáveis ou de Hurwitz. O interesse por estes polinômios começou com o estudo da teoria de estabilidade.

O estudo da teoria de estabilidade está intrinsecamente relacionado com a análise dos mecanismos de controle por retro-alimentação. De fato a teoria da estabilidade foi estimulada pela necessidade de resolução de problemas técnicos advindos dos controladores automáticos que apareceram na revolução industrial. Um tipo de controlador por retro-alimentação é o mecanismo conhecido como o controlador - ou regulador- de Watt [10], que apareceu em torno de 1788. No início, estes mecanismos funcionaram bem, fazendo com que os motores mantivessem a velocidade de rotação desejada. O estado ideal do motor pode ser visto como um ponto de equilíbrio do sistema. Como os controladores faziam o sistema voltar para o estado ideal quando houvesse pequenas perturbações, tal estado podia ser visto como um ponto de equilíbrio estável. Daí vem o forte relacionamento entre a estabilidade do sistema e a teoria de controle por retro-alimentação.

Com o passar do tempo, houve melhorias nos materiais usados na fabricação dos controladores. Paradoxalmente, esta melhoria acabou causando o mau funcionamento dos controladores. Maxwell 
explicou o motivo desta perda de estabilidade em "On Governors" [11], tratando cada caso dos vários tipos de controladores, resolvendo as equações linearizadas dos sistemas e analisando as condições específicas dos parâmetros físicos do sistema para que as raízes dos polinômios característicos das ditas linearizações tivessem parte real negativa. Com isto Maxwell demonstrou que as raízes com parte real negativa do polinômio característico do sistema fornecem a condição que decide a estabilidade. Daí vem o termo "polinômio estável"e a importância destes no estudo da estabilidade. O problema estudado por Maxwell foi aperfeiçoado por Routh [17] em 1877. Independentemente de Routh, em 1895 Hurwitz publicou um artigo [8] que resolveu o problema com algumas generalizações. Cabe observar, entretanto, que as condições de estabilidade de um polinômio foi estudado do ponto de vista matemático por C. Hermite em 1856.

A pesquisa nesta área é chamada atualmente de controle robusto. Uma discussão mais detalhada sobre o contexto histórico e teorias que apareceram nesta área pode ser visto em [13].

Na prática, pensando em cada coeficiente dos polinômios como parâmetro de controle, temos uma família de sistemas cujos parâmetros estão num intervalo limitado determinado pelos limites físicos do modelo do sistema. Os resultados de Routh e de Hurwitz tratam de um único polinômio característico da família. Como nos problemas de controle por retro-alimentação os parâmetros de controle variam toda vez que aparece um novo valor de entrada, teremos que verificar se o sistema, com os novos parâmetros, é estável.

Um exemplo prático deste tipo de problema é fornecido por um ônibus automático percorrendo um caminho conhecido previamente. A massa total do ônibus varia em razão da mudança do número de passageiros. O coeficiente de atrito varia dependendo das condições da estrada a ser percorrida (inclinações, curvatura) e das condições meteorológicas (dia claro, dia chuvoso). É possível acontecer a perda de estabilidade (acidente) quando começa a chover, mesmo com o sistema sendo estável em dias sem chuva. Logo, toda vez que o coeficiente de atrito muda, teremos que aplicar novamente os 
critérios de estabilidade.

Para resolver problemas deste tipo, podemos fazer uma análise das bifurcações tal como em [19], definindo o local onde há a mudanças de estabilidade, ou podemos encontrar um critério de estabilidade válido para toda a família de polinômios. Na segunda linha de possibilidade a extensão dos resultados de Routh e Hurwitz foi desenvolvida por V.L. Kharitonov em [9].

Neste trabalho, apresentaremos os critérios de Routh, Hurwitz e Kharitonov e daremos um exemplo de aplicação a um problema na área de controle robusto. 


\section{Capítulo 2}

\section{Preliminares}

Daremos a seguir algumas definições e resultados sobre estabilidade no sentido de Liapunov e apresentaremos a motivação para estudar os polinômios estáveis.

\subsection{Estabilidade de Liapunov}

Sejam $\Omega$ aberto em $\mathbb{R} \times \mathbb{R}^{n}$ e $f$ uma função contínua de $\Omega$ em $\mathbb{R}^{n}$. Consideramos o seguinte sistema

$$
\grave{x}^{\prime}=f(t, x)
$$

Definição 2.1.1 Seja $\varphi(t)$ uma órbita de 2.1 definida em $[0, \infty), \varphi(t)$ é estável se para todo $\varepsilon>0$ existe $\delta>0$ tal que se $\psi(t)$ é solução de (2.1) e $\left|\psi\left(t_{0}\right)-\varphi\left(t_{0}\right)\right|<\delta$ então $\psi$ está definida para qualquer $t \geq t_{0} e|\psi(t)-\varphi(t)|<\varepsilon$ para qualquer $t \geq t_{0}$. Se além disso existir $\delta_{1}>0$ tal que $\left|\psi\left(t_{0}\right)-\varphi\left(t_{0}\right)\right|<\delta_{1}$ implica $\lim _{t \rightarrow \infty}|\psi(t)-\varphi(t)|=0$ então $\varphi(t)$ é dita assintoticamente estável.

Neste trabalho, estaremos mais interessados em órbitas em torno de um ponto de equilíbrio $x_{0}$ do sistema (2.1). 
Definição 2.1.2 Seja $x_{0}$ um ponto de equilíbrio de (2.1). Diz-se que $x_{0}$ é estável no sentido de Liapunov se para qualquer $\varepsilon>0$ e qualquer $t_{0}>0$, existe $\delta>0$ tal que para qualquer $x_{1} \in B_{\delta}\left(x_{0}\right)$, a solução $\varphi$ de $\dot{x}=f(t, x), x\left(t_{0}\right)=x_{1}$, é definida em $\left[t_{0}, \infty\right)$ e $\left|\varphi(t)-x_{0}\right|<\varepsilon$ para todo $t$ em $\left[t_{0}, \infty\right)$. Se além disso existe $\delta_{1}>0$ tal que $x_{1} \in B_{\delta_{1}}\left(x_{0}\right)$ implica $\lim _{t \rightarrow \infty}\left|\varphi(t)-x_{0}\right|=0$, $x_{0}$ é dito assintoticamente estável.

\subsection{Equações lineares com coeficientes constantes}

Consideramos o sistema

$$
\dot{x}=A x
$$

onde $A$ é um operador linear em $\mathbb{R}^{n}$. Neste caso, a solução é dada por:

$$
\varphi(t, x)=e^{t A} x
$$

Por causa disso, o comportamento de trajetórias tem forte relação com os valores próprios de $A$. Em particular, se $A$ tem todos os valores próprios com parte real negativa foi provado em [20] que $x_{0}$ é estável assintoticamente.

Teorema 2.2.1 Todos os valores próprios de A têm parte real negativa se e somente se, existem $\mu>0$ e $k \geq 1$ tais que

$$
\left|e^{t A} x\right| \leq k e^{-\mu t}|x|
$$

para todo $x \in \mathbb{R}^{n}$ e $t \geq 0$.

Claro que este teorema garante estabilidade assintótica em torno de 0. Observe que este resultado é global, isto é, começando em qualquer ponto em $\mathbb{R}^{n}$, a trajetória deste ponto converge para origem quando $t \rightarrow \infty$. Abrindo mão deste propriedade global, podemos melhorar o resultado para uma 
classe mais geral de funções.

Teorema 2.2.2 Consideramos o sistema quase-linear

$$
\dot{x}=A x+g(t, x),(t, x) \in \Omega_{b}
$$

onde $\Omega_{b}=\left\{(t, x) \in \mathbb{R} \times \mathbb{R}^{n}|| x \mid<b\right\}$, A é um operador linear em $\mathbb{R}^{n}$ cujos autovalores têm parte real negativa, $g$ é uma função continua que satisfaz $\lim _{|x| \rightarrow 0}|g(t, x)|=0$ uniformemente em t. Se supusermos que (2.5) tem solução única em todo ponto, então a solução nula de (2.5) é assintoticamente estável.

Em particular, podemos aplicar o teorema 2.2 .2 ao sistema autônomo

$$
\dot{x}=f(x)
$$

onde $f: \Omega \subset \mathbb{R}^{n}$ é $C^{1}$ e $\Omega \in \mathbb{R}^{n}$ é aberto.

Corolário 2.2.3 Seja $x_{0}$ um ponto singular do sistema (2.6) e suponhamos que $D f\left(x_{0}\right)$ tem todos os autovalores com parte real negativa. Então existe uma vizinhança $U$ de $x_{0}$ e constantes $k>0$, $\mu>0$ tais que para todo $x \in U$, a solução $\varphi(t)$ de (2.6) tal que $\varphi(0)=x$ está definida para todo $t \geq 0$ $e\left|\varphi(t)-x_{0}\right| \leq k e^{-\mu t}\left|x-x_{0}\right|$ para qualquer $t \geq 0$. Em particular, $x_{0}$ é assintoticamente estável.

Observe que a hipótese essencial nos dois teoremas acima é todos os valores próprios de $A$ terem parte real negativa. Estes resultados reduzem o problema de estabilidade dos sistemas (2.2), (2.5) e (2.6) a um problema puramente algébrico: verificar se o polinômio característico do problema tem todas as raízes com parte real negativa. Note que devemos verificar o polinômio característico de $A$ em (2.2) e (2.5) e verificar o polinômio característico de $D f\left(x_{0}\right)$ em (2.6). 
Definição 2.2.4 Um polinômio P é chamado de Hurwitz (ou estável) se, e somente se, todas as suas raízes têm parte real negativa. Isto é, para qualquer $z \in \mathbb{C}, p(z)=0$ implica Rez $<0$.

Este é o motivo natural de ser chamado de polinômio estável. Nos próximos capítulos, apresentaremos critérios para caracterizar os polinômios de Hurwitz. 


\section{Capítulo 3}

\section{Critério de estabilidade}

Neste capítulo nosso objetivo será apresentar critérios para decidir se um polinômio com coeficientes reais tem todas suas raízes com parte real negativa, isto é, quando ele é de Hurwitz. Seguiremos a apresentação feita em [5]. Trabalharemos aqui com coeficientes reais. Usaremos a seguinte notação: $\mathbf{i}=\sqrt{-1}$.

\subsection{Propriedades dos polinômios de Hurwitz}

Apresentaremos a seguir algumas propriedades dos polinômios de Hurwitz, definição 2.2.4.

Consideremos um polinômio

$$
P(z)=a_{0} z^{n}+a_{1} z^{n-1}+\ldots+a_{n}
$$

onde $a_{s} \in \mathbb{R}, s \in 0,1,2, \ldots, n$, e $a_{0} \neq 0$.

\subsubsection{Condição necessária}

Apresentaremos uma condição necessária para um polinômio ser de Hurwitz. 
Proposição 3.1.1 Para (3.1) ser polinômio de Hurwitz é necessário que as desigualdades

$$
\frac{a_{1}}{a_{0}}>0, \frac{a_{2}}{a_{0}}>0, \ldots, \frac{a_{n-1}}{a_{0}}>0, \frac{a_{n}}{a_{0}}>0
$$

sejam satisfeitas.

Demonstração Como o polinômio possui somente coeficientes reais, as suas raízes são reais ou pares de raízes complexas conjugadas. Denotaremos as raízes reais por $r_{s}, s=1,2, \ldots, p$, e as raízes complexas por $z_{t}, \widetilde{z}_{t}, t=1,2, \ldots, q \operatorname{com} q=\frac{n-p}{2}$. Então podemos escrever $P(z)$ pela seguinte expressão

$$
\begin{gathered}
P(z)=a_{0} \prod_{s=1}^{p}\left(z-r_{s}\right) \prod_{t=1}^{q}\left(z-z_{t}\right)\left(z-\widetilde{z}_{t}\right), \\
P(z)=a_{0} \prod_{s=1}^{p}\left(z-r_{s}\right) \prod_{t=1}^{q}\left(z^{2}-2 R e z_{t} z+\left|z_{t}\right|^{2}\right) .
\end{gathered}
$$

Como $P(z)$ é polinômio de Hurwitz, $r_{s}<0$ para todo $s$ e $R e z_{t}<0$ para todo $t$. Logo, todos os coeficientes de $\frac{P(z)}{a_{0}}$ são produtos de elementos positivos, o que implica as desigualdades de (3.2)

Observe que $-\frac{a_{1}}{a_{0}}$ é a soma das raízes. O fato de $\frac{a_{1}}{a_{0}}>0$ implica que o polinômio $P$ tem pelo menos uma raiz com parte real negativa. A condição necessária exclui os polinômios que possuem todas as raízes com parte real positiva.

Para um polinômio de grau igual a dois,

$$
P(z)=a_{0} z^{2}+a_{1} z+a_{2}
$$

as desigualdades (3.2) constituem uma condição suficiente para $P(z)$ ser estável. Neste caso, $z=$ $\frac{-a_{1} \pm \sqrt{a_{1}^{2}-4 a_{0} a_{2}}}{2 a_{0}} ;$ se $4 a_{0} a_{2}>a_{1}^{2}$, a parte real de $z$ é $\frac{-a_{1}}{2 a_{0}}<0$. No caso contrário, como $\frac{a_{1}}{2 a_{0}}>$ 
$\sqrt{\left(\frac{a_{1}}{2 a_{0}}\right)^{2}-\frac{a_{2}}{a_{0}}}, \frac{-a_{1}}{2 a_{0}}$ decide o sinal de $z$, isto é $z<0$. Logo $P(z)$ é de Hurwitz.

Entretanto para um polinômio de grau maior ou igual a 3, as desigualdades (3.2) já não são condições suficientes. Por exemplo,

$$
P(z)=z^{3}+z^{2}+z+1=(z+1)\left(z^{2}+1\right)
$$

não é um polinômio de Hurwitz, pois as raízes são $-1, \mathbf{i},-\mathbf{i}$.

\subsubsection{Condição geométrica}

Para obtermos as condições necessárias e suficientes, vamos analisar o comportamento geométrico de um polinômio de Hurwitz. Para isso, representaremos o polinômio obtido substituindo $z$ por $\mathbf{i} w$ em $P(z)$, onde $w \in \mathbb{R}$. Assim os termos de grau par de $P(\mathbf{i} w)$ contribuem com a parte real e os termos de grau ímpar contribuem com a parte imaginária, possibilitando a análise do comportamento de $P(\mathbf{i} w)$ no plano complexo. Com a substituição de $\mathbf{i} w$, podemos desenhar a imagem de $P(\mathbf{i} w)$ no plano complexo. Vejamos alguns exemplos.

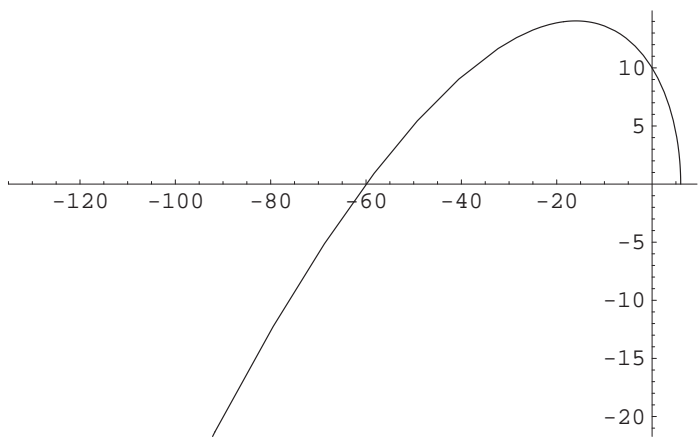

Figura 3.1: $P_{1}(\mathbf{i} w)=(\mathbf{i} w)^{3}+6(\mathbf{i} w)^{2}+11(\mathbf{i} w)+6=(\mathbf{i} w+1)(\mathbf{i} w+2)(\mathbf{i} w+3)$

Na figura 3.1, o polinômio $P_{1}$ tem três raízes negativas. Quando $w$ varia de 0 a $+\infty, P_{1}(\mathbf{i} w)$ 


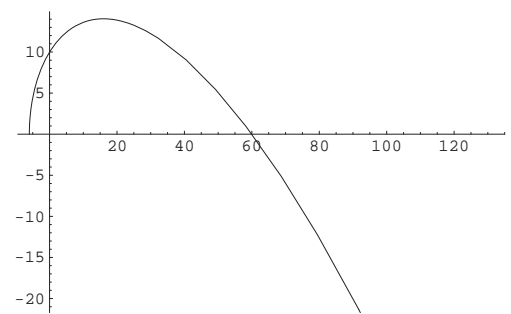

Figura 3.2: $P_{2}(\mathbf{i} w)=(\mathbf{i} w)^{3}-6(\mathbf{i} w)^{2}+11(\mathbf{i} w)-6=(\mathbf{i} w-1)(\mathbf{i} w-2)(\mathbf{i} w-3)$

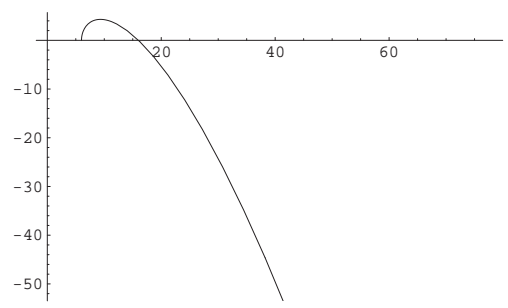

Figura 3.3: $P_{3}(\mathbf{i} w)=(\mathbf{i} w)^{3}-2(\mathbf{i} w)^{2}-5(\mathbf{i} w)+6=(\mathbf{i} w-1)(\mathbf{i} w+2)(\mathbf{i} w-3)$

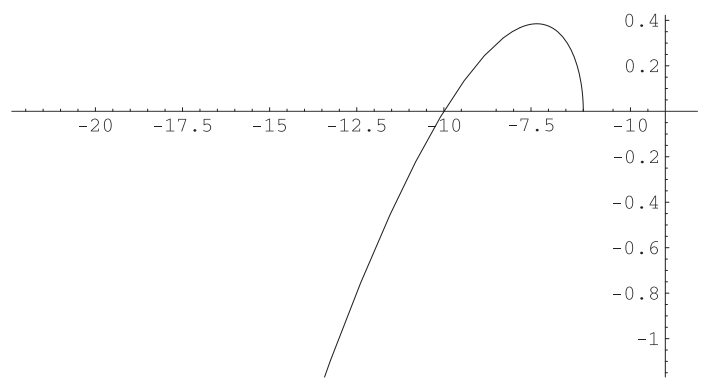

Figura 3.4: $P_{4}(\mathbf{i} w)=(\mathbf{i} w)^{3}+4(\mathbf{i} w)^{2}+(\mathbf{i} w)-6=(\mathbf{i} w-1)(\mathbf{i} w+2)(\mathbf{i} w+3)$

percorre três quadrantes no sentido anti-horário. Comparemos com uma função que só tem raízes positivas, que é o caso da figura 3.2 onde $P_{2}$ tem três raízes positivas. $P_{2}(\mathbf{i} w)$ percorre três quadrantes no sentido horário.

Na figura $3.3, P_{3}$ tem duas raízes positivas e uma negativa, $P(\mathbf{i} w)$ passa diretamente do primeiro 


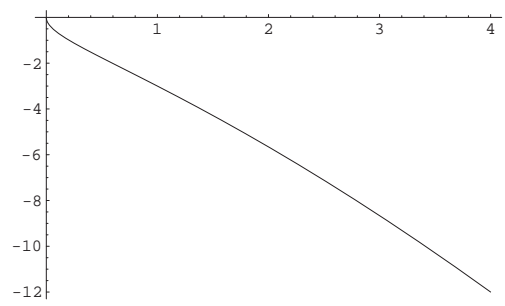

Figura 3.5: $P_{5}(\mathbf{i} w)=(\mathbf{i} w)^{3}-(\mathbf{i} w)^{2}-2(\mathbf{i} w)=(\mathbf{i} w)(\mathbf{i} w+1)(\mathbf{i} w-2)$

ao quarto quadrante no sentido horário. Já com uma raíz positiva e duas negativas, figura $3.4, P_{4}(\mathbf{i} w)$ se desloca no sentido anti-horário, cruzando um eixo apenas a partir do segundo quadrante.

Se uma raíz é 0 e as outras duas são raízes de sinais opostos (figura 3.5 ), $P_{5}(\mathbf{i} w)$ começa na origem e não cruza nenhum eixo, permanecendo no mesmo quadrante.

Com estes dados, podemos supor que o sinal das raízes e o número das quais são positivas e das quais são negativas afetam o sentido da trajetória assim como o número de quadrantes que $P(\mathbf{i} w)$ percorre. Para formalizar isso, vamos analisar o ângulo percorrido por $P(\mathbf{i} w)$ quando $w$ varia de 0 a $+\infty$

Proposição 3.1.2 Se $P(z)$ é polinômio de Hurwitz com grau $n \geq 1$, então $\arg (P(\mathbf{i} w)), w \in(0,+\infty)$, é contínua e estritamente crescente em $w$.

Demonstração Sem perda de generalidade, consideremos $a_{0}=1 . P(z)$ é polinômio de Hurwitz. Se $z_{k}$, com $k=1,2, \ldots, n$, são as raízes de $P(z)$, então

$$
P(z)=\prod_{k=1}^{n}\left(z-z_{k}\right),
$$


onde $z_{k}=\alpha_{k}+\mathbf{i} \beta_{k}, \alpha_{k}, \beta_{k} \in \mathbb{R}$ e $\alpha_{k}<0$ para todo $k$. Em particular,

$$
P(z)=\prod_{k=1}^{n}\left(z+\left|\alpha_{k}\right|-\mathbf{i} \beta_{k}\right) .
$$

Como o argumento do produto é a soma dos argumentos dos fatores,

$$
\arg (P(\mathbf{i} w))=\sum_{k=1}^{n} \arg \left(\mathbf{i} w+\left|\alpha_{k}\right|-\mathbf{i} \beta_{k}\right) .
$$

O argumento é dado pelo arco-tangente. É fácil compreendermos esta equação analisando a figura 3.6. Como o polinômio é de Hurwitz, não há nenhum $\alpha_{k}=0$, então todos os arcos-tangente estão bem definidos. A altura é $\left(w-\beta_{k}\right)$ e a base é $\left|\alpha_{k}\right|$.

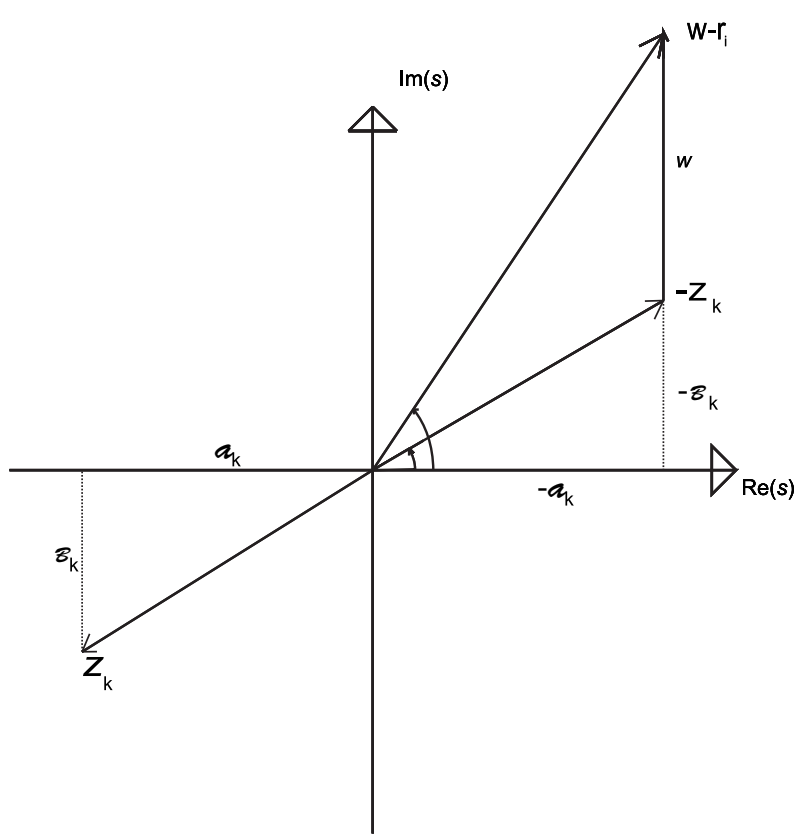

Figura 3.6: Tangente para as raízes com parte real negativa 


$$
\arg (P(\mathbf{i} w))=\sum_{k=1}^{n} \arctan \left(\frac{w-\beta_{k}}{\left|\alpha_{k}\right|}\right)
$$

Como o arco-tangente é estritamente crescente em $\frac{w-\beta_{k}}{\left|\alpha_{k}\right|}$ e $\frac{w-\beta_{k}}{\left|\alpha_{k}\right|}$ cresce quando $w$ cresce, $\arctan \left(\frac{w-\beta_{k}}{\left|\alpha_{k}\right|}\right)$ é estritamente crescente em $w$ para todo $k$. Isso também é fácil de ver na figura 3.7. Conseqüentemente $\arg (P(\mathbf{i} w))$, que é a soma dos arcos-tangentes, também é estritamente crescente e ainda, como arco-tangente é contínua, $\arctan \left(\frac{w-\beta_{k}}{\left|\alpha_{k}\right|}\right)$ também é contínua.

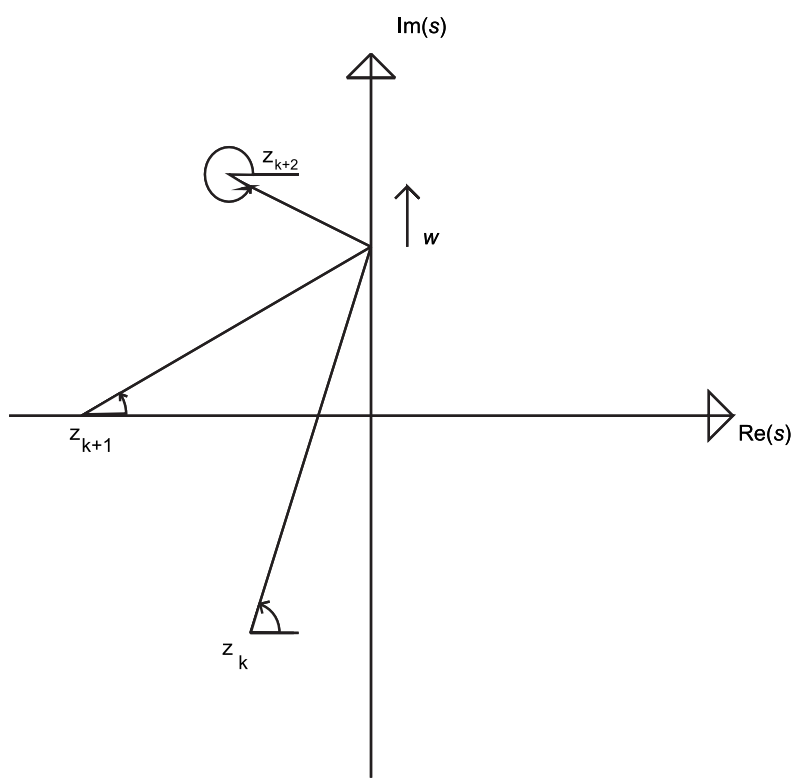

Figura 3.7: Montonicidade estritamente crescente de $\arg (P(\mathbf{i} w))$

Observe que usamos fortemente o fato de $P(z)$ ser polinômio de Hurwitz. A expressão (3.9) se dá pelo fato das raízes terem parte real negativa, como ilustrado na figura 3.6.

Se $P(z)$ tem somente raízes com parte real positiva, $\arg (P(\mathbf{i} w))$ será uma função contínua e estritamente decrescente; $P(\mathbf{i} w)$ percorre o plano no sentido anti-horário como a figura 3.2 . 
De fato, se as raízes tiverem somente parte real positiva, a equação fica da seguinte forma.

$$
\arg (P(\mathbf{i} w))=\sum_{k=1}^{n} \arg \left(\mathbf{i} w-\left|\alpha_{k}\right|-\mathbf{i} \beta_{k}\right)
$$

Se $w=0$, cada (i $\left.w-\left|\alpha_{k}\right|-\mathbf{i} \beta_{k}\right), k=1,2, \ldots, n$ está no lado esquerdo do eixo imaginário. Quando $w$ crescer, o ângulo entre o vetor $\left(\mathbf{i} w-\widetilde{\left|\alpha_{k}\right|}-\mathbf{i} \beta_{k}\right)$ e o eixo $x$ tende a $\frac{\pi}{2}$, ou seja $P(\mathbf{i} w)$ percorre o plano no sentido horário, ficando estritamente decrescente, como ilustrado nas figuras $3.2 \mathrm{e} 3.8$.

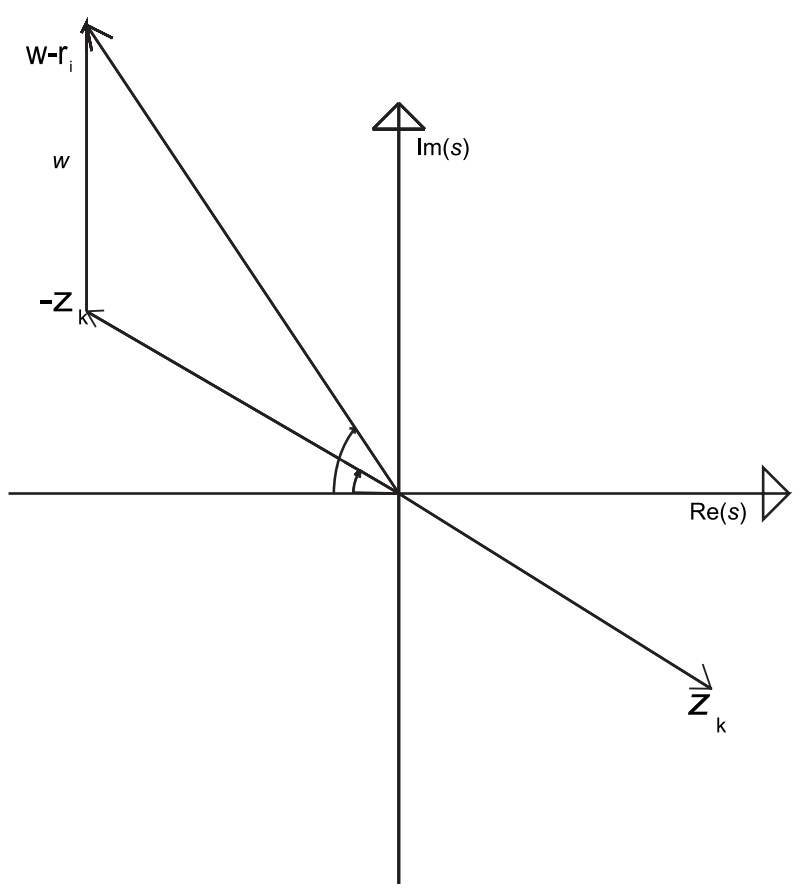

Figura 3.8: Tangente para as raízes com parte real positiva

Se $\arg (P(z))$ é bem definido e contínuo em $w$, isto é $P(\mathbf{i} w) \neq 0$ para qualquer $w$, podemos definir

$$
\operatorname{Arg}(P)=\lim _{w \rightarrow \infty}(\arg (P(\mathbf{i} w))-\arg (P(0)))
$$


onde a expressão denota o ângulo percorrido por $P(\mathbf{i} w)$ quando $w$ varia de 0 a $+\infty$. Subtrair $\arg (P(0))$ é como colocar o ponto inicial em cima do eixo real positivo. No caso de raiz com parte real negativa, quando $w$ cresce, $P(i w)$ tende ao eixo imaginário positivamente. Já no caso de raiz com parte real positiva, tende ao eixo imaginário negativamente. Observar a figura 3.6 e $\underline{3.8}$. Então, cada raiz de parte real negativa contribui com $\frac{\pi}{2}$ para $\operatorname{Arg}(P)$, cada raiz de parte real positiva contribui com $-\frac{\pi}{2}$. Se existe algum $w_{r}$ tal que $P\left(\mathbf{i} w_{r}\right)=0$, o argumento fica indeterminado. Assim temos

Proposição 3.1.3 Um polinômio de grau n é de Hurwitz se, e somente se, $\operatorname{Arg}(P)$ é bem definido e contínuo $(P(\mathbf{i} w) \neq 0$ para qualquer $w)$ e igual a $\frac{n \pi}{2}$.

Demonstração Se $P$ é de Hurwitz, vale a expressão (3.10). Como $P$ não possui raízes puramente imaginárias, $\operatorname{Arg}(P)$ é bem definido. Usando a expressão (3.12), temos $\operatorname{Arg}(P)=\frac{n \pi}{2}$.

Provemos na outra direção. Se $\operatorname{Arg}(P)$ é bem definido, $P(\mathbf{i} w) \neq 0$, ou seja, não tem raízes puramente imaginárias. $\operatorname{Arg}(P)=\frac{n \pi}{2}$ significa que existem pelo menos $n$ raízes com parte real negativa. Como $P$ é de grau $n$, todas as raízes terão a parte real negativa. Logo $P$ é de Hurwitz.

Observe que se $P$ é de Hurwitz, quando $w$ varia de 0 a $+\infty, P(\mathbf{i} w)$ sempre percorre o plano complexo no sentido anti-horário, pois $\arg (P(\mathbf{i} w))$ é estritamente crescente e ainda percorre $n$ quadrantes, pois $\operatorname{Arg}(P)=\frac{n \pi}{2}$.

Esta analise de $\operatorname{Arg}(P)$ é uma simplificação de índice de Cauchy. Ver [2] e [21].

A figura 3.1 é um exemplo imediato. O polinômio possui 3 raízes negativas com grau $3 . \arg (P(\mathbf{i} w))$ é contínuo, $\operatorname{Arg}(P)$ é bem definido e é igual a $\frac{3 \pi}{2}$, percorrendo o primeiro, segundo e terceiro quadrantes.

Vimos acima o critério geométrico para caracterizar os polinômios de Hurwitz. Mas temos que analisar o gráfico de $P(\mathbf{i} w)$ ou verificar se o valor de $\operatorname{Arg}(P)=\frac{n \pi}{2}$. Isto é, verificar se $P(\mathbf{i} w)$ percorreu 
$n$ quadrantes. Para simplificar este critério, relacionaremos os valores de $w$ onde $P(\mathbf{i} w)$ cruza o eixos real e imaginário com a função que parametriza a "trajetória" de $P(\mathbf{i} w)$ quando $w$ varia de 0 a $+\infty$.

Como $P(\mathbf{i} w)$ tem somente coeficientes reais, podemos expressá-lo da seguinte maneira, usando dois polinômios reais $P_{1}$ e $P_{2}$ :

$$
P(\mathbf{i} w)=P_{1}\left(w^{2}\right)+\mathbf{i} w P_{2}\left(w^{2}\right)
$$

onde o grau de $P_{1}$ é $\left[\frac{n}{2}\right]$ e o grau de $P_{2}$ é $\left[\frac{n-1}{2}\right]$. Assim podemos desenhar o gráfico de $P(\mathbf{i} w)$ no plano complexo. $P_{1}\left(w^{2}\right)$ é o valor no eixo real, $w P_{2}\left(w^{2}\right)$ é o valor no eixo imaginário. Observe que se $w \neq 0$, as raízes de $P_{2}(w)$ e $w P_{2}(w)$ são as mesmas. Já sabemos que a parte imaginária de $P(i 0)$ é nula. Logo, basta analisar as raízes de $P_{1}$ e $P_{2}$.

Se $n=2 k$, o grau de $P_{1}$ é $k$, de $P_{2}$ é $k-1$. Se $n=2 k+1$, o grau de ambos é $k$.

Definição 3.1.4 Chamamos de diagrama de resposta de Leonhard a imagem de $P(\mathbf{i} w)$ onde $w$ varia de 0 ate $\infty$.

Exemplos de diagrama de resposta de Leonhard já vimos nas figuras 3.1, 3.2, 3.3 e 3.4 .

Pela Proposição 3.1.3, se $P$ for de Hurwitz, $P(\mathbf{i} w)$ percorrerá $n$ quadrantes. Isso significa que $P_{1}$ de (3.13) se anula ao passar do primeiro para o segundo quadrante, enquanto $P_{2}$ mantém seu sinal. Isto é, $P_{2}$ não se anula no primeiro quadrante. $P_{2}$ se anula ao $P(\mathbf{i} w)$ passar do segundo quadrante para terceiro enquanto $P_{1}$ mantém seu sinal. Os zeros $z_{l m}$, com $l=1,2$ e $m=1,2, \ldots,\left[\frac{n}{2}\right]$, dos polinômios $P_{1}, P_{2}$ correspondem aos valores de $w^{2}$ onde $P(\mathbf{i} w)$ intercepta os eixos do plano complexo. No caso de $P$ ser de Hurwitz, as raízes de formato $w^{2}$ têm que serem reais e aparecem em ordem crescente. Logo os $z_{l m}$ têm que serem todos positivos e as raízes de $P_{1}$ e $P_{2}$ têm que serem alternados entre si.

$$
0<z_{11}<z_{21}<z_{12}<z_{22}<z_{13}<z_{23}<z_{14}<\cdots
$$


Se isto não ocorrer será porque $P(\mathbf{i} w)$ deslocou-se no sentido contrário (i.é. horário) pelo menos uma vez, não percorrendo portanto os $n$ quadrantes. Observe também que $P(\mathbf{i} w) \neq 0$, pois $P_{1}$ e $P_{2}$ nunca se anulam simultaneamente.

De fato, se a desigualdade (3.14) for satisfeita por $P$, não ocorrem casos de polinômios com raízes de sinais diferentes, como nas figuras $3.3,3.4,3.5$.

No entanto, não excluímos o caso que todas as raízes terem parte real positiva. Só com a desigualdade (3.14) não podemos garantir a estabilidade do polinômio. Um exemplo é dado na figura 3.2. Ela só tem as raízes com parte real positiva, mas satisfaz a desigualdade (3.14). Falta a garantia de $P(\mathbf{i} w)$ percorrer o plano no sentido anti-horário.

Para consertar isso, ou precisamos analisar a mudança de sinais em torno dos zeros de $P_{1}$ e $P_{2}$ (verificar se $P_{1}$ começou positivo, e quando $P_{1}$ se anula pela primeira vez, verificar que $P_{2}$ é positivo, etc.), ou simplesmente excluir o caso dos polinômios com todas as raízes com parte real positiva, colocando na hipótese que $P$ satisfaz a condição necessária.

Proposição 3.1.5 O polinômio $P(z)$ é de Hurwitz se, somente se, os zeros dos polinômios $P_{1}$ e $P_{2}$ definidos em (3.13) são reais, satisfazem as desigualdades (3.14) e as desigualdades (3.2).

Demonstração Se $P(z)$ é estável, é imediato. Claro que $P_{1}$ e $P_{2}$ são reais. $P(\mathbf{i} w)$ percorre $n$ quadrantes. Logo satisfazem as desigualdades (3.14).

Provemos a recíproca. As desigualdades (3.14) excluem o caso de polinômios que têm simultaneamente raízes com parte real positiva e negativa, pois se tal fato ocorrer, $P(\mathbf{i} w)$ não pode percorrer $n$ quadrantes quando $w$ varia de 0 até $+\infty$. Entretanto as desigualdades (3.14) garantem que $P(\mathbf{i} w)$ percorre $n$ quadrantes. E a condição necessária garante que não ocorre o caso de polinômio com todas as raízes com parte real positiva. Assim, só sobra o caso de polinômio com todas as raízes com 
parte real negativa.

A proposição 3.1 .5 é uma das várias caracterizações para polinômio estável. Em particular, é uma variação de teorema de Herimite-Biehler que apresenta uma serie de condições equivalentes para polinômio estável. Ver [7], [2], [16] e [21].

A proposição 3.1.5 será útil na demonstração de critérios algébricos.

\subsection{Critérios de Routh e Hurwitz}

Exigimos, sem perda de generalidade, que na expressão (3.1) $a_{0}$ é positivo. Mostraremos a seguir uma condição necessária e suficiente para que $P(z)$ seja polinômio de Hurwitz.

Primeiro apresentaremos o nosso principal resultado, neste capítulo, que é o critério de Routh.

Teorema 3.2.1 O polinômio $P(z)$ é de Hurwitz se, e somente se, as desigualdades

$$
c_{1, s}>0,
$$

para $s=1,2,3, \ldots, n$, e as desigualdades (3.2) são satisfeitas. 
onde os $c_{p, q}$ são definidas pelo seguinte esquema.

$$
\begin{array}{c|ccc} 
& c_{1,0}=a_{0}, & c_{2,0}=a_{2}, & c_{3,0}=a_{4}, \ldots \\
& c_{1,1}=a_{1}, & c_{3,1}=a_{5}, \ldots \\
r_{2}=\frac{a_{0}}{a_{1}} & c_{2,1}=a_{3}, & c_{3,2}=c_{4,0}-r_{2} c_{4,1}, \ldots \\
r_{3}=\frac{c_{1,1}}{c_{1,2}}=c_{2,0}-r_{2} c_{2,1}, & c_{2,2}=c_{3,0}-r_{2} c_{3,1}, & c_{3,3}=c_{4,1}-r_{3} c_{4,2}, \ldots \\
\hline r_{q}=\frac{c_{1, q-2}}{c_{1, q-1}} & c_{1,3}=c_{2,1}-r_{3} c_{2,2}, & c_{2,3}=c_{3,1}-r_{3} c_{3,2}, & \\
& \multicolumn{3}{c}{c_{p, q}=c_{p+1, q-2}-r_{q} c_{p+1, q-1}, \ldots} \\
c_{1, n}=a_{n}
\end{array}
$$

onde $p=1,2,3, \ldots$ e $q=2,3,4, \ldots$.

Os passos que seguiremos para demonstrar este teorema são os seguintes:

1) Usamos a equivalência da proposição 3.1.5.

2) Com 1), o nosso objetivo se reduziu a verificar se o polinômio $P$ tiver grau $n=2 k$ (respectivamente $n=2 k+1), P_{1}$ tem $k$ (respectivamente $k$ ) raízes e $P_{2}$ tem $k-1$ (respectivamente $k$ ) raízes. E ainda temos que verificar se as raízes satisfazem as desigualdades (3.14).

3) Para verificar 2), estudaremos como foi construído o esquema de Routh.

4) Construiremos funções que fazem o papel de $P_{1}$ e $P_{2}$ de (3.13) a partir do polinômio $P$. Para isso, criaremos uma seqüência de polinômios, usando o método ou Algoritmo de Euclides. Dito método se assemelha à construção do esquema de Routh, a menos de sinal.

5)A condição (3.15) fornece as propriedades corretas para verificar as condições dadas em 2).

A verificação de condições impostas em 2) é o nosso principal objetivo.

Primeiro visualizemos melhor como foi criado este esquema. 


\subsubsection{Construção de esquema de Routh}

Formamos uma matriz criada a partir dos coeficientes de polinômio. Suponhamos que $n=2 k$ para algum $k$ natural.

$$
\left(\begin{array}{ccccccc}
a_{1} & a_{0} & 0 & 0 & \ldots & 0 & 0 \\
a_{3} & a_{2} & a_{1} & a_{0} & \ldots & 0 & 0 \\
a_{5} & a_{4} & a_{3} & a_{2} & \ldots & 0 & 0 \\
\ldots & \ldots & \ldots & \ldots & \ldots & \ldots & \ldots \\
a_{n-1} & a_{n-2} & a_{n-3} & a_{n-4} & \ldots & \ldots & \ldots \\
0 & a_{n} & a_{n-1} & a_{n-2} & \ldots & \ldots & \ldots \\
\ldots & \ldots & \ldots & \ldots & \ldots & \ldots & \ldots \\
0 & 0 & 0 & 0 & \ldots & a_{n-1} & a_{n-2} \\
0 & 0 & 0 & 0 & \ldots & 0 & a_{n}
\end{array}\right)
$$

(3.17) é uma matriz $n \times n$. Para $0<2 i-j<n$, o elemento geral $a_{i j}=a_{2 i-j}$. 
Se $n$ for ímpar,

$$
\left(\begin{array}{ccccccc}
a_{1} & a_{0} & 0 & 0 & \ldots & 0 & 0 \\
a_{3} & a_{2} & a_{1} & a_{0} & \ldots & 0 & 0 \\
a_{5} & a_{4} & a_{3} & a_{2} & \ldots & 0 & 0 \\
\ldots & \ldots & \ldots & \ldots & \ldots & \ldots & \ldots \\
a_{n-2} & a_{n-3} & a_{n-4} & a_{n-5} & \ldots & \ldots & \ldots \\
a_{n} & a_{n-1} & a_{n-2} & a_{n-3} & \ldots & \ldots & \ldots \\
\ldots & \ldots & \ldots & \ldots & \ldots & \ldots & \ldots \\
0 & 0 & 0 & 0 & \ldots & a_{n-1} & a_{n-2} \\
0 & 0 & 0 & 0 & \ldots & 0 & a_{n}
\end{array}\right)
$$

Nas operações posteriores, voltemos a considerar $P \operatorname{com} n=2 k$ (Vamos apresentar caso par, caso ímpar alternadamente). Observe que a primeira coluna de (3.17) equivale a linha $c_{i, 1}$ de (3.16) e a segunda coluna equivale à linha $c_{i, 0}$. Subtraímos as colunas $1,3,5, \ldots$ multiplicadas por $r_{2}=\frac{a_{0}}{a_{1}}$ das 
colunas $2,4, \ldots$

$$
\left(\begin{array}{ccccccc}
a_{1} & 0 & 0 & 0 & \ldots & 0 & 0 \\
a_{3} & a_{2}-r_{2} a_{3} & a_{1} & 0 & \ldots & 0 & 0 \\
a_{5} & a_{4}-r_{2} a_{5} & a_{3} & a_{2}-r_{2} a_{3} & \ldots & 0 & 0 \\
\ldots & \ldots & \ldots & \ldots & \ldots & \ldots & \ldots \\
a_{n-1} & a_{n-2}-r_{2} a_{n-1} & a_{n-3} & a_{n-4}-r_{2} a_{n-3} & \ldots & \ldots & \ldots \\
0 & a_{n} & a_{n-1} & a_{n-2}-r_{2} a_{n-2} & \ldots & \ldots & \ldots \\
\ldots & \ldots & \ldots & \ldots & \ldots & \ldots & \ldots \\
0 & 0 & 0 & 0 & \ldots & a_{n-1} & a_{n-2}-r_{2} a_{n-1} \\
0 & 0 & 0 & 0 & \ldots & 0 & a_{n}
\end{array}\right)
$$

Observe que a segunda coluna, ignorando o zero da primeira linha, de (3.19) é $c_{i, 2}$ do esquema (3.16). Em seguida, subtraímos a coluna $2 l$, onde $l$ é $1,2,3,4, \ldots$, multiplicada por $r_{3}=\frac{a_{1}}{a_{2}-r_{2} a_{3}}$, das colunas 
$2 l+1$.

$$
\left(\begin{array}{ccccccc}
a_{1} & 0 & 0 & 0 & \ldots & 0 & 0 \\
a_{3} & c_{1,2} & 0 & 0 & \ldots & 0 & 0 \\
a_{5} & c_{2,2} & a_{3}-r_{3} c_{2,2} & c_{1,2} & \ldots & 0 & 0 \\
\ldots & \ldots & \ldots & \ldots & \ldots & \ldots & \ldots \\
a_{n-1} & c_{k, 2} & a_{n-3}-r_{3} c_{k, 2} & c_{k-1,2} & \ldots & \ldots & \ldots \\
0 & a_{n} & a_{n-1}-r_{3} a_{n} & c_{k, 2} & \ldots & \ldots & \ldots \\
\ldots & \ldots & \ldots & \ldots & \ldots & \ldots & \ldots \\
0 & 0 & 0 & 0 & \ldots & a_{n-1}-r_{3} a_{n} & c_{k, 2} \\
0 & 0 & 0 & 0 & \ldots & 0 & a_{n}
\end{array}\right)
$$

Novamente a terceira coluna, ignorando os zeros de primeira e segunda linha, equivale à $c_{l, 3}$ do esquema de Routh. Continuando esta operação até que todos termos em cima da diagonal principal sejam 0, temos:

$$
\left(\begin{array}{ccccccc}
a_{1} & 0 & 0 & 0 & \ldots & 0 & 0 \\
a_{3} & c_{1,2} & 0 & 0 & \ldots & 0 & 0 \\
a_{5} & c_{2,2} & c_{1,3} & 0 & \ldots & 0 & 0 \\
\ldots & \ldots & \ldots & \ldots & \ldots & \ldots & \ldots \\
a_{n-1} & c_{k, 2} & c_{k-1,3} & c_{k-2,4} & \ldots & \ldots & \ldots \\
0 & a_{n} & c_{k, 3} & c_{k-1,4} & \ldots & \ldots & \ldots \\
\ldots & \ldots & \ldots & \ldots & \ldots & \ldots & \ldots \\
0 & 0 & 0 & 0 & \ldots & c_{1, n-1} & 0 \\
0 & 0 & 0 & 0 & \ldots & 0 & a_{n}
\end{array}\right)
$$


que é exatamente esquema de Routh "transposto" (ignorando os zeros em cima de diagonal principal).

É fácil ver que se chamarmos de $H_{i}$ a matriz $i \times i$, onde $i=1,2, \ldots, n$, formados por $a_{p, q}$ de (3.17) onde $p, q \leq i$,

$\operatorname{det} H_{1}=c_{1,1}, \operatorname{det} H_{2}=c_{1,1} c_{1,2}, \operatorname{det} H_{3}=c_{1,1} c_{1,2} c_{1,3}, \ldots, \operatorname{det} H_{n}=c_{1,1} c_{1,2} c_{1,3} c_{1,4} \ldots c_{1, n}$.

\subsubsection{Construção de uma seqüência de polinômios a partir de esquema de Hurwitz}

Vamos construir funções que fazem o papel de $P_{1}$ e $P_{2}$ de (3.13) a partir do polinômio $P$ e usá-las na proposição 3.1.5. Para esta construção, definiremos os polinômios que têm os mesmos coeficientes do $c_{l, 0}$ e $c_{l, 1}$, onde $l=1,2,3, \ldots$

$$
h_{1}(z)=\frac{1}{2}(P(z)+P(-z)), h_{2}(z)=\frac{1}{2}(P(z)-P(-z))
$$

Primeiro, consideremos que o grau de $P(z)$ é par, $n=2 k$. Neste caso, $h_{1}$ só tem termos de grau par, $h_{2}$ tem termos de grau ímpar. Se o grau de $P(z)$ for $n=2 k+1$, trocamos as definições acima por:

$$
h_{1}(z)=\frac{1}{2}(P(z)-P(-z)), h_{2}(z)=\frac{1}{2}(P(z)+P(-z))
$$

Voltemos para o polinômio de grau $n=2 k$,

$$
\begin{gathered}
h_{1}(z)=a_{0} z^{n}+a_{2} z^{n-2}+a_{4} z^{n-4}+\ldots+a_{n} \\
h_{2}(z)=a_{1} z^{n-1}+a_{3} z^{n-3}+a_{5} z^{n-5}+\ldots+a_{n-1} z
\end{gathered}
$$


Aplicando o método de Euclides, também denominado Algoritmo de Euclides, para polinômios, que é um algoritmo para achar o máximo divisor comum de $h_{1}$ e $h_{2}$, construímos uma seqüência de funções $h_{i}$, onde $i=1,2,3, \ldots, n$

$$
h_{i}(z)=\widetilde{r_{i+1}} z h_{i+1}(z)-h_{i+2}(z)
$$

Se encontrarmos algum $l$ tal que $h_{l}$ seja zero, o máximo divisor comum será $h_{l-1}$. O algoritmo mostrado acima é um pouco diferente do algoritmo usual de Euclides para polinômios devido ao aparecimento de $z$ no primeiro termo do lado direito da equação (3.27). O motivo desta diferença é que o grau de $h_{l}$ é um grau maior do que grau de $h_{l+1}$ para todo $l$. E se $H_{l}$ só tiver termos de grau par, $h_{l+1}$ só tem termos de grau ímpar.

Observe que esta operação (3.27) é parecida com a criação da matriz (3.21). A diferença aparece devido ao último termo de (3.27) ter sinal negativo. O que resulta:

$$
\widetilde{r_{i}}=-1^{i} r_{i}
$$

para $i=2,3, \ldots, n$, alternando o sinal de $r_{i}$ de (3.16).

Proposição 3.2.2 Se o polinômio $P(z)$ é de Hurwitz, então $h_{i}$ não é identicamente nulo para todo $i=1,2,3, \ldots$.

Demonstração Suponha, por absurdo, que exista $h_{i} \equiv 0$, ou seja, $h_{1}$ e $h_{2}$ têm divisor comum não trivial. Seja $d$ o divisor comum entre $h_{1}$ e $h_{2}$, então:

$$
h_{1}(z)=\frac{1}{2}(P(z)+P(-z))=l_{1}(z) d(z)
$$




$$
\begin{gathered}
h_{2}(z)=\frac{1}{2}(P(z)+P(-z))=l_{2}(z) d(z) \\
P(z)=\left(l_{1}(z)+l_{2}(z)\right) d(z) \\
P(-z)=\left(l_{1}(z)-l_{2}(z)\right) d(z)
\end{gathered}
$$

$d(z)$ também é divisor comum entre $P(z)$ e $P(-z)$. Lembrando a expressão (3.4), podemos concluir que o divisor comum $d$ tem que ter o formato de $\left(z^{2}+c\right)$ onde $c$ é alguma constante real. O termo $\left(z-r_{s}\right)$ de (3.4) não pode ser o divisor comum entre $P(-z)$ e $P(z)$, restando os termos de tipo $\left(z^{2}-2 R e z_{t} z+\left|z_{t}\right|^{2}\right)$. Mas o termo $2 R e z_{t} z$ muda de sinal quando $z$ muda de sinal então concluímos que $R e z_{t}=0$. Logo, $P(z)$ não é polinômio de Hurwitz, o que contradiz a hipótese.

Para chegar no formato de $P_{1}$ e $P_{2}$ de (3.13), definiremos uma seqüência de polinômios $g_{i}$, onde $i=1,2,3, \ldots$, a partir de $h_{i}$. Consideremos que $P(z)$ tem grau $n=2 k$, onde $k$ é natural.

$$
\begin{gathered}
g_{1}(z)=a_{0} z^{k}+a_{2} z^{k-1}+\cdots+a_{2 k} . \\
g_{2}(z)=a_{1} z^{k-1}+a_{3} z^{k-2}+\cdots+a_{2 k-1} .
\end{gathered}
$$

$g_{1}$ e $g_{2}$ coincidem com $P_{1}$ e $P_{2}$ de (3.13), exceto pelo sinal dos coeficientes, devido ao número imaginário usado em (3.13). Mais precisamente, a relação entre $g_{l}$ e $P_{l}$ é:

$$
P_{l}(u)=g_{l}(-u)
$$


para $l=1,2$.

Esta relação não afeta a análise das raízes. A única diferença é que $P_{l}$ tem as raízes reais positivas enquanto $g_{l}$ tem as raízes reais negativas para $l=1,2$. O resto da seqüência $g_{i}$ é definido por:

$$
h_{2 i-1}(s) \doteq g_{2 i-1}\left(s^{2}\right), h_{2 i}(s) \doteq s g_{2 i}\left(s^{2}\right), i=1, \ldots, k
$$

Se $P(z)$ tiver grau ímpar, $n=2 k+1$, usamos as definições de $h_{1}$ e $h_{2}$ de (3.24) e definimos

$$
h_{2 i+1}(s) \doteq s g_{2 i+1}\left(s^{2}\right), h_{2 i}(s) \doteq g_{2 i}\left(s^{2}\right), i=1, \ldots, k .
$$

Substituindo $g_{2 i-1}$ e $g_{2 i}$ de (3.36) na equação (3.27), temos a seguinte relação:

$$
\begin{gathered}
g_{2 i+1}(z)=\widetilde{r_{2 i}} z g_{2 i}(z)-g_{2 i-1}(z) \\
g_{2 i+2}(z)=\widetilde{r_{2 i+1}} g_{2 i+1}(z)-g_{2 i}(z)
\end{gathered}
$$

Finalmente achamos os polinômios equivalentes a $P_{1}$ e $P_{2}$ de (3.13). Vamos observar algumas propriedades de $g_{i}$, a partir de (3.38) e (3.39).

Lema 3.2.3 Seja $P(z)$ é um polinômio de Hurwitz, $g_{i}$ a seqüência dos polinômios criados a partir de P. Se $\widetilde{z}$ é zero de $g_{i}, \operatorname{sinal}\left(g_{i-1}\right) \neq \operatorname{sinal}\left(g_{i+1}\right)$ para $i=2,3,4, \ldots$

Demonstração Sejam $\widetilde{z_{l}}$ os zeros de $g_{l}$, para $l=1,2,3,4, \ldots$ Se $l$ for par, substituímos $i=\frac{l}{2}$ na 
equação (3.38). Se $l$ for ímpar, substituímos $i=\frac{l-1}{2}$ na equação (3.39). É fácil ver que:

$$
g_{i+1}(\widetilde{z})=-g_{i-1}(\widetilde{z})
$$

Em outras palavras, enquanto $\operatorname{sinal}\left(g_{i-1}\right)=\operatorname{sinal}\left(g_{i}+1\right), g_{i}$ não se anula para $i=2,3,4, \ldots$ Mais precisamente, numa seqüência de ternas $\left\{g_{i-1}, g_{i}, g_{i+1}\right\}$, não há a raíz do $g_{i}$ no caso das seguintes triplas de sinais:

$$
\begin{aligned}
& \{+++\} \\
& \{+-+\} \\
& \{-\quad+\quad-\} \\
& \{-\quad-\quad-\}
\end{aligned}
$$

$g_{i}$ só pode anular-se no caso de triplas tais como:

$$
\begin{aligned}
& \{+\quad+-\} \\
& \{-++\} \\
& \{-\quad-+\} \\
& \{+-\quad-\}
\end{aligned}
$$

quando há exatamente uma troca de sinais.

Como trabalhamos com os polinômio de Hurwitz que satisfazem a condição necessária para polinômios estáveis, excluímos os casos que se tem zeros para $g_{i}$ e $g_{i+1}$ ao mesmo tempo, pois se ocorrer isto, pela relação (3.40), $g_{i-1}$ e $g_{i+2}$ também se anulam. Este processo continua até $g_{1}$ e $g_{n}$, mas $g_{n}$ é uma constante que não é zero pela condição necessária $\left(g_{n}=a_{n}\right)$. O caso de $g_{i-1}(\widetilde{z})=g_{i+1}(\widetilde{z})=0$ 


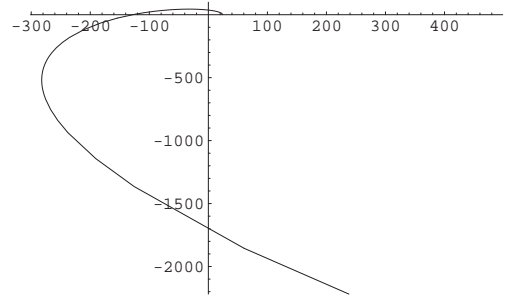

Figura 3.9: diagrama de Leonhard para $\mathrm{P}(\mathrm{z})$

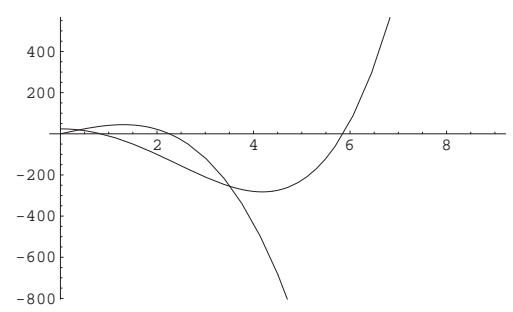

Figura 3.10: $P_{1}(z)$ e $z P_{2}(z)$

também não pode ocorrer, pois as expressões (3.38) e (3.39), obriga $g_{i}(\widetilde{z})=0$, caindo no caso dos zeros consecutivos.

Observe que $g_{i}$ formam uma seqüência de Sturm. Ver [2] e [21].

Vamos ver um exemplo desta seqüência.

\section{Exemplo 3.2.4}

$$
P(z)=z^{4}+10^{3}+35^{2}+50 z+24
$$

Observe que este polinômio é de Hurwitz.

Como podemos observar na figura $3.9 \mathrm{e}$ 3.10. $P(\mathbf{i} w)$ percorre 4 quadrantes no sentido anti-horário e ainda as raízes de $P_{1}$ e $P_{2}$ são reais e alternadas. 
O esquema de Routh é:

$$
\left(\begin{array}{cccc}
10 & 0 & 0 & 0 \\
50 & 30 & 0 & 0 \\
0 & 24 & 42 & 0 \\
0 & 0 & 0 & 24
\end{array}\right)
$$

$$
\begin{aligned}
& h_{1}(z)=z^{4}+35 z^{2}+24 \\
& h_{2}(z)=10 z^{3}+50 z \\
& h_{3}(z)=z^{4}+5 z^{2}-\left(z^{4}+35 z^{2}+24\right)=-30 z^{2}-24 \\
& h_{4}(z)=-1 \times\left(-10 z^{3}-8 z\right)-\left(10 z^{4}+50 z\right)=-42 z \\
& g_{1}(x)=x^{2}+35 x+24 \\
& g_{2}(x)=10 x+50 \\
& g_{3}(x)=-30 x-24 \\
& g_{4}(x)=-42
\end{aligned}
$$




\begin{tabular}{c|cccc}
$x=-\infty$ & $g_{1}(x)$ & $g_{2}(x)$ & $g_{3}(x)$ & $g_{4}(x)$ \\
\hline$\frac{-35-\sqrt{1129}}{2}$ & + & - & + & - \\
& 0 & - & + & - \\
-5 & - & - & + & - \\
& - & 0 & + & - \\
-0.8 & - & + & 0 & - \\
& - & + & - & - \\
$\frac{-35+\sqrt{1129}}{2}$ & 0 & + & - & - \\
\hline$x=+\infty$ & + & + & - & -
\end{tabular}

As raízes de $g_{1}$ são $\frac{-35 \pm \sqrt{1129}}{2}$, de $g_{2}$ é -5 , de $g_{3}$ é -0.8 . A tabela (3.53) apresenta a análise dos sinais de $g_{i}(x)$, variando $\mathrm{x}$ de $-\infty$ a $+\infty$ para determinar como é o comportamento perto de raízes de cada $g_{i}(x)$. Os sinais são alternados para $x$ suficientemente negativo. Como já discutimos, os $g_{2}(x)$ e $g_{3}(x)$ não podem ser nulos simultaneamente. $g_{4}$ é uma constante. O primeiro que se anula é o $g_{1}$. Após a troca de sinal de $g_{1}$, o $g_{2}$ da tripla $\left\{g_{1}, g_{2}, g_{3}\right\}$ é permitido anular-se. Este fenômeno é importante, pois é daqui que tiramos o argumento para obter o número de raízes de $g_{1}$ e $g_{2}$ e as desigualdades entre as raízes.

Lema 3.2.5 Seja $P(z)$ um polinômio com grau $n=2 k$ que satisfaz a desigualdade (3.2) $e$ a desigualdade

$$
c_{1,1}>0, c_{1,2}>0, c_{1,3}>0, \ldots, c_{1, n}>0 .
$$

Então $g_{1}(z)$ tem $k$ raízes reais negativas, $g_{2}(z)$ tem $k-1$ raízes reais negativas. Se $P(z)$ tiver grau $n=2 k+1, g_{1}(z)$ e $g_{2}(z)$ terão $k$ raízes reais negativas. 
Demonstração Como as desigualdades (3.2) estão satisfeitas, $r_{i}$ são todos positivos, o sinal de $\widetilde{r_{i}}$ se alternam. Lembrando as operações das matrizes para formar a esquema de Routh, os sinais de coeficientes do termo de maior grau de $g_{i}, i=1,2,3, \ldots$, são

$$
+,+,-,-,+,+,-,-,+,+,-,-, \ldots
$$

Esta seqüência de sinais é devida à escolha do sinal de último termo de (3.27) como negativo. Observe as seguintes equações.

$$
h_{1}-\widetilde{r_{2}} z h_{2}=-h_{3}
$$

O coeficiente do termo de maior grau de $-h_{3}$ é $c_{13}>0$ (primeiro termo da terceira linha da matriz (3.17)). Como o sinal do coeficiente do termo de maior grau de $h_{i}$ é igual ao sinal do coeficiente do termo de maior grau de $g_{i}$, o sinal do coeficiente do termo de maior grau de $g_{3}$ fica negativo.

$$
h_{2}-\widetilde{r_{3}} z h_{3}=h_{2}-r_{3}\left(-h_{3}\right)=-h_{4}
$$

O coeficiente do termo de maior grau de $-h_{4}$ é $c_{1,4}>0$, o sinal do coeficiente de termo de maior grau de $g_{4}$ fica negativo. Na próxima vez,

$$
h_{3}-\widetilde{r_{4}} z h_{4}=h_{3}-r_{4} z h_{4}=-\left(-h_{3}-r_{4}\left(-h_{4}\right)\right)=-h_{5}
$$

O coeficiente do termo de maior grau de $h_{5}$ é $c_{1,5}$. O sinal do coeficiente do termo de maior grau de $g_{5}$ é positivo. Assim foi construída a seqüência de sinais (3.55). 
O grau dos polinômios $g_{i}, i=1,2,3, \ldots, k$ são

$$
k, k-1, k-1, k-2, k-2, k-3, k-3, \ldots, 1,1,0
$$

Isso é fácil de ver, pois o primeiro termo do lado direito de equação (3.38), ao ser multiplicado por $z$ aumenta um grau, e o termo de maior grau do $\widetilde{r_{2 i}} z g_{2 i}(z)$ e do $g_{2 i-1}(z)$ se cancelam, ou seja, $g_{2 i+1}$ mantém o mesmo grau de $g_{2 i}$. No caso da equação (3.39), só é cancelado o termo de maior grau, assim $g_{2 i+2}$ decai um grau.

Agora, fixemos $z,-\infty<z<\infty$ e criamos uma seqüência

$$
g_{1}(z), g_{2}(z), g_{3}(z), \ldots, g_{2 k}(z)
$$

Seja $T(z)$ o número de troca de sinais desta seqüência $g_{i}(z), i=1,2, \ldots, 2 k-1$ ( $g_{2 k}$ é um constante). Para $z>0$ suficientemente grande, os sinais dos $g_{i}$ são os mesmos da seqüência (3.55). Os sinais são trocadas dois a dois. Isto é, $T(\infty)=k$. Para $z<0$ suficientemente negativo, os sinais se alternam. $T(\infty)=2 k$

Então $T(-\infty)-T(\infty)=k$ sempre, ou seja, quando $z$ varia de $-\infty$ para $\infty$, perdemos $k$ troca de sinais. Claro que os locais onde são perdidas as trocas de sinais são os zeros do $g_{i}$.

Afirmamos que tal perda só pode ocorrer nos zeros de $g_{1}$. Seja $\widetilde{z_{i}}$ é zero de $g_{i}$. Analisaremos a troca de sinais de $g_{i}$ em $\widetilde{z_{i}}, 1<i<2 k-1$. Lembremos o lema 3.2 .3 e as ternas (3.42) a que são permitidos terem raízes. Todos os tipos de ternas que permitem a $g_{i}, i=2,3,4, \ldots$ ter raízes, no máximo tem uma troca de sinais. Quando $z=\widetilde{z_{i}}, g_{i}(z)$ se anula, mas o número de troca de sinais se mantem. Logo não há perda do número de trocas de sinais nos zeros de $g_{i}$, onde $1<i<2 k$. Então $k$ perdas do número de trocas sobram para as raízes de $g_{1}$. Como $g_{1}$ tem $k$ perdas de trocas, tem $k$ 
raízes. Estas raízes são reais porque variamos $z$ de $-\infty$ para $\infty$ somente na reta real. E ainda como $P$ só tem coeficientes positivos, $g_{1}$ também só tem coeficientes positivos, o que leva a concluir que as raízes de $g_{1}$ são negativas.

A demonstração para $g_{2}$ é análoga. Consideramos $g_{i}(z)$ onde $i=2,3, \ldots, 2 k$, temos $T(-\infty)-$ $T(\infty)=k-1$, pois, para $z$ suficientemente negativo tem-se $2 k-1$ trocas de sinais, enquanto para $z$ suficientemente positivo tem-se $k$ trocas de sinais. Pela mesma razão apresentada acima, para zeros de $g_{i}, 2<i<2 k$ não se tem perda de troca de sinal. Logo $g_{2}$ tem $k-1$ raízes reais negativas.

Para o caso das $n=2 k+1$, depois de fazer as alterações adequadas anteriormente comentadas de $h_{i}$ e $g_{i}$, os sinais dos coeficientes dominantes continuam tendo a mesma seqüência do que vimos em (3.55) (Isso vem da construção da seqüência de $h_{i}$ ). Mas o grau de polinômios $g_{i}, i=1,2,3, \ldots, k$ mudam para:

$$
k, k, k-1, k-1, k-2, k-2, k-3, \ldots, 1,1,0
$$

Assim quando $z$ é suficientemente negativo, todos os sinais serão os mesmos (Se $k$ é ímpar, $g_{i}$ é negativo para todo $i=1,2,3, \ldots$ Se $k$ é par, é positivo). A partir daqui, usa-se exatamente o mesmo argumento que usamos no caso par, aplicando o lema 3.2.3. Só nas extremidades pode ter-se a perda de troca de sinais. A única diferença é que $g_{1}$ e $g_{2}$ têm $k$ raízes reais negativas.

Lema 3.2.6 Seja $P(z)$ um polinômio que satisfaz a desigualdade (3.2) e a desigualdade (3.54). Seja a seqüência $g_{i}$ criada a partir de $P$ pela construção acima. Então as raízes de $g_{1}$ e $g_{2}$ são intercaladas entre si. Isso é, entre duas raízes de $g_{1}$ existe exatamente uma raiz de $g_{2}$ (exceto extremidades no caso de $n$ impar).

Demonstração Pelo lema 3.2.5, já sabemos que $g_{1}$ tem $k$ raízes reais negativas e que $g_{1}$ perde $k$ trocas de sinais. Afirmo que entre todos os $g_{i}$, o menor zero é de $g_{1}$. Vimos no lema anterior, se $z$ 
é negativo suficientemente, ou a seqüência $g_{i}$ tem os sinais alternados ou tem todos os sinais iguais. Já sabemos que neste tipo de seqüência, $g_{i}$ que estão no "meio"não podem zerar. Neste caso, o candidato a anular são as extremidades da caso seqüência. Um lado é constante. O único polinômio que é permitido ter a raíz nesta configuração de sinais é $g_{1}$. Após da troca do sinal de $g_{1}$, as possíveis seqüências são

$$
\{-,-,+,-,+,-, \ldots\},\{-,+,+,+, \ldots\},\{+,-,-,-, \ldots\}
$$

Nesta situação, os únicos $g_{i}$ que podem anular-se são $g_{1}$ e $g_{2}$. Se, ao aumentar $z, g_{2}$ não tiver raíz até encontrar o próximo zero de $g_{1}, g_{1}$ não perde o número da troca de sinais da seqüência (Volta para o estado que era antes). O $g_{1}$ só tem $k-1$ raízes, portanto nesta situação, o máximo de troca de sinais que ele perde é $k-1$, o que contraria o lema 3.2.5. Ou seja, o resultado do lema 3.2.5 obriga as raízes de $g_{1}$ a se intercalarem com as raízes de $g_{2}$.

De certa maneira, para manter o número de trocas que foi estabelecido pelo lema 3.2.5, a perda da troca de sinal que aparece em $g_{1}$ tem que se "propagar" para o lado direito da seqüência antes de aparecer o próximo zero de $g_{1}$. Se seguirmos o raciocínio que usamos para achar o número de raízes de $g_{1}$ e $g_{2}$ para outros $g_{i}$, i=3, $4,5, \ldots$, todas as raízes entre $g_{i}$ e $g_{i+1}, \mathrm{i}=2,3,4, \ldots, \mathrm{n}-1$ serão intercaladas. A "propagação" de perda de troca de sinais pára exatamente antes de $g_{n}$, que é constante. Já sabemos que $g_{1}$ e $g_{2}$ são os polinômios que são equivalentes a $P_{1}$ e $P_{2}$ de (3.13).

\subsubsection{Teorema de Routh e de Hurwitz}

Relembremos o critério de Routh.

Teorema 3.2.7 O polinômio $P(z)$ é de Hurwitz se, e somente se, as desigualdades (3.2) e as desigualdades (3.54) são satisfeitas. 
Demonstração Primeiro, suponha que o grau de $P(z)$ é par e satisfaz as desigualdades (3.2) e (3.54). Pelo lema 3.2.5 e 3.2.6, temos $g_{1}$ com $k$ raízes reais negativas e $g_{2}$ com $k-1$ raízes reais negativas que são intercaladas entre si. Mas isso é equivalente à desigualdade de (3.14). Lembrando que vale (3.35), é satisfeita a condição da proposição 3.1.5 Logo $P(z)$ é de polinômio de Hurwitz.

Por outro lado, se $P(z)$ é polinômio de Hurwitz, pela proposição 3.1 .5 as raízes de $g_{1}$ e $g_{2}$ são intercaladas (satisfaz (3.14)), reais e negativas. De qualquer maneira, isso não ocorre se $P(z)$ não satisfizer $c_{1, i}>0$ para todo $i$. Pois se algum $c_{1, i}$ for 0 ou negativo, perdemos o número de troca de sinais adequadas.

\section{Exemplo 3.2.8}

$$
P(z)=z^{5}+15 z^{4}+85 z^{3}+225 z^{2}+274 z+120
$$

Temos um exemplo de aplicação imediata.

Criamos o esquema de Routh.

$$
\left(\begin{array}{cccc} 
& 1 & 85 & 274 \\
& 15 & 225 & 120 \\
\frac{1}{15} & 60 & 266 & \\
\frac{1}{4} & \frac{317}{2} & 120 & \\
\frac{120}{317} & \frac{82882}{317} & & \\
\frac{5389}{165764} & 24 & &
\end{array}\right)
$$

Os coeficientes de $P(z)$ são positivos e $c_{1, i}$ são positivos também. $P(z)$ é polinômio de Hurwitz.

\section{Exemplo 3.2.9}


Peguemos o polinômio (3.6).

$$
P(z)=z^{3}+z^{2}+z+1
$$

É um polinômio que satisfaz condição necessária, mas não é polinômio de Hurwitz. Vamos ver o esquema de Hurwitz deste polinômio. A matriz fica da seguinte forma

$$
\left(\begin{array}{lll}
1 & 0 & 0 \\
1 & 0 & 1 \\
0 & 0 & 1
\end{array}\right)
$$

Como $c_{1,2}=0$, não podemos completar o esquema. De qualquer maneira, como $c_{i, 2}=0, P$ não é o polinômio de Hurwitz (Não podemos criar $h_{i}$ até $h_{2 k+1}$ e não temos suficiente número da trocas de sinal).

Existe um teorema que não utiliza o esquema de Hurwitz, embora utilize os mesmos cálculos. Apresentamos um teorema equivalente ao critério de Routh, que é o teorema de Hurwitz. Consideremos a matriz (3.17) e os comentários feitos no item (3.22).

Teorema 3.2.10 O polinômio $P(z)$ é de Hurwitz se, e somente se, as desigualdades de (3.2) e as desigualdades

$$
\operatorname{det} H_{1}>0, \operatorname{det} H_{2}>0, \operatorname{det} H_{3}>0, \ldots, \operatorname{det} H_{n}>0 \text {. }
$$

são satisfeitas.

Demonstração A prova é imediata das operações que foram realizadas com matrizes e de (3.22).

Observe que para polinômio $P(z)$ de qualquer grau, a condição necessária para $P(z)$ ser polinômio de Hurwitz já garante $a_{i}>0$, em particular, det $H_{1}>0$, e pela propriedade de determinantes, $\operatorname{det} H_{n-1}=a_{n} \operatorname{det} H_{n}$, ou seja, sinal de $\operatorname{det} H_{n-1}$ é igual ao sinal de $\operatorname{det} H_{n}$. Isto diminui calculo do 
exemplo abaixo.

\section{Exemplo 3.2.11}

Se um polinômio $P(z)$ tiver grau 2, a condição necessária para $P(z)$ ser de Hurwitz é $a_{0}>0, a_{1}>0$ e $a_{2}>0$. A condição suficiente é $a_{1}>0$ e $a_{1} a_{2}>0$, que já é satisfeita pela condição necessária.

Se um polinômio $P(z)$ tiver grau 3 , a condição necessária nos dá a informação de que det $H_{1}>0$, o sinal det $H_{3}$ é mesmo de $\operatorname{det} H_{2}$, então o que resta verificar é

$$
\operatorname{det} H_{2}=a_{1} a_{2}-a_{0} a_{3}>0
$$

Se valer a desigualdade (3.68), $P(z)$ é polinômio de Hurwitz. Para caso de $n=4$, a condição suficiente é dada pela desigualdade $(3.68)$ e

$$
\operatorname{det} H_{3}=a_{1} a_{2} a_{3}-a_{0} a_{3}^{2}-a_{1}^{2} a_{4}>0
$$

Na verdade, a desigualdade (3.68) é redundante, pois

$$
\begin{array}{r}
a_{3}\left(a_{1} a_{2}-a_{0} a_{3}\right)-a_{1}^{2} a_{4}>0 \\
\left(a_{1} a_{2}-a_{0} a_{3}\right)>\frac{a_{1}^{2} a_{4}}{a_{3}}>0
\end{array}
$$

Logo se $P(z)$ satisfizer a desigualdade (3.69) com $a_{i}>0$ para todo $i$, a desigualdade (3.68) também é satisfeita. Suponha que $P(z)$ satisfaz a desigualdade (3.68). Então se $\widetilde{P(z)}$ é um outro polinômio de grau 3

$$
\widetilde{P(z)}=\widetilde{a_{0}} z^{3}+\widetilde{a_{1}} z^{2}+\widetilde{a_{2}} z+\widetilde{a_{3}}
$$




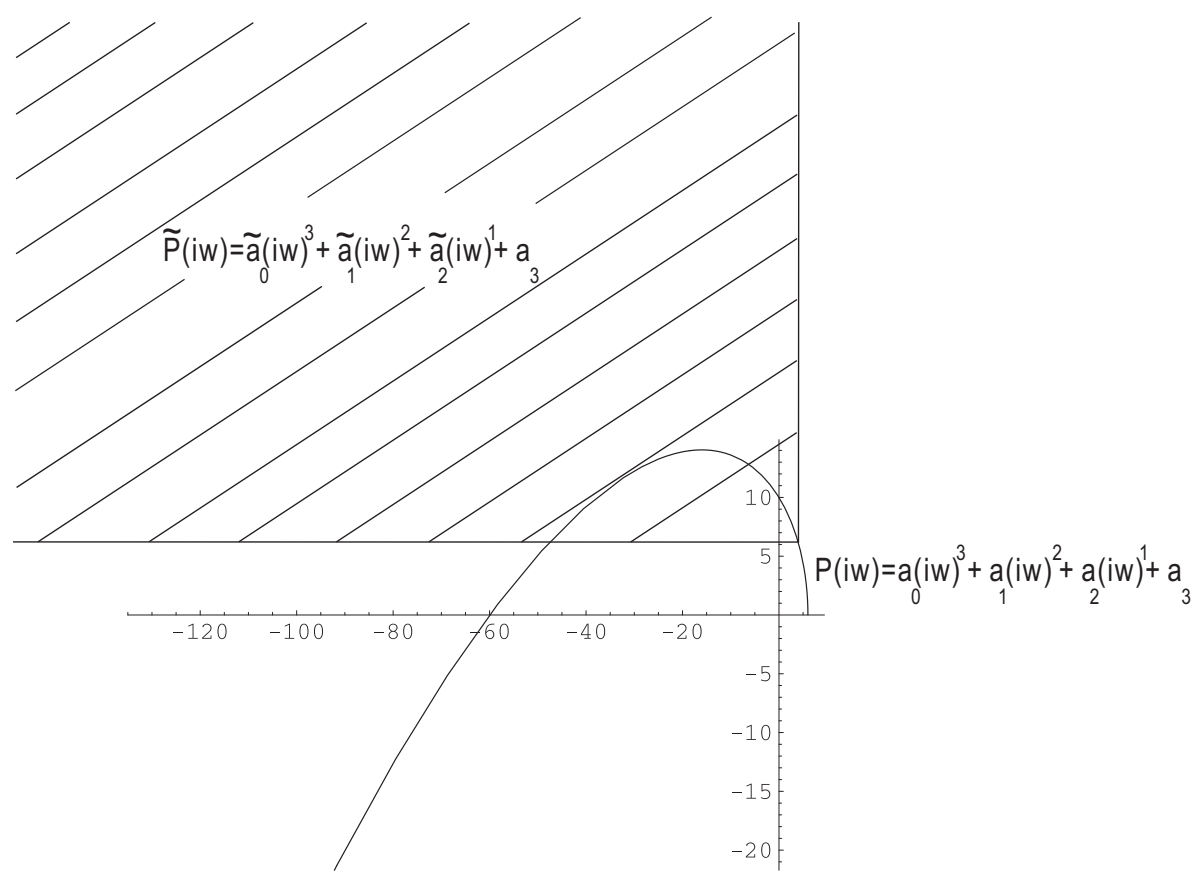

Figura 3.11: A região definida pelas desigualdades (3.73) no plano complexo

tal que vale as seguintes desigualdades:

$$
\widetilde{a_{1}} \geq a_{1}, \widetilde{a_{2}} \geq a_{2}, 0<\widetilde{a_{3}} \leq a_{3}, 0<\widetilde{a_{0}} \leq a_{0},
$$

$\widetilde{P(z)}$ também é polinômio de Hurwitz. Pois se chamarmos de $\widetilde{H}_{i}$, das desigualdades $(3.73)$ e (3.68) resulta $\operatorname{det} \widetilde{H_{2}}>0$, o que é condição suficiente para $\widetilde{P(z)}$ ser polinômio de Hurwitz.

Vejamos o diagrama de Leonhard deste polinômio.

$$
\widetilde{P(\mathbf{i} w)}=-\widetilde{a_{0}} \mathbf{i} w^{3}-\widetilde{a_{1}} w^{2}+\widetilde{a_{2}} \mathbf{i} w+\widetilde{a_{3}}
$$


A região hachurada da figura 3.11 é onde ficam os polinômios que satisfazem as desigualdades (3.73) para algum $\mathbf{i} w$. Quando $w$ varia para $+\infty$, a região hachurada acompanha a imagem de $P(\mathbf{i} w)$ mantendo seus limites paralelos aos eixos real e imaginário.)

Este fato nos sugere uma possibilidade de achar muitos polinômios estáveis a partir de poucos testes de estabilidade. Se pudermos generalizar isto, será um resultado interessante, pois poderemos verificar a estabilidade de uma família de polinômios com poucos testes. No próximo capítulo, generalizaremos para polinômios de grau arbitrário de o que fizemos para exemplos de grau 3. 


\section{Capítulo 4}

\section{Teorema de Kharitonov}

Neste capítulo, o objetivo será apresentar uma generalização do que vimos no exemplo 3.2.11. As demonstrações de [4], [14, [1] foram consideradas.

\subsection{Teorema de Kharitonov}

Nesta seção, vamos definir uma família $K$ de polinômios e apresentaremos um critério para que todos os polinômios desta família sejam de Hurwitz.

\subsubsection{Teorema de Kharitonov}

Fixemos um número natural $n>0$ e $a_{l}{ }^{-}, a_{l}{ }^{+} \in \mathbb{R}^{n}, a_{l}{ }^{-} \leq a_{l}{ }^{+}$para todo $l=0,1,2,3, \ldots, n-1$. Definimos um conjunto $K$ de polinômios de grau n tal que

$$
P(z)=z^{n}+a_{n-1} z^{n-1}+\ldots+a_{0}
$$

onde $a_{l} \in\left[a_{l}{ }^{-}, a_{l}{ }^{+}\right]$para todo $l=0,1,2,3, \ldots, n-1$.

Supomos sem perda de generalidade, que o coeficiente do termo de maior grau de $P(z)$ é igual a 
um. Observe que, diferentemente de capítulo anterior, invertemos a ordem dos índices dos coeficientes do polinômio para facilitar as definições de alguns polinômios que apresentaremos a seguir.

Definiremos quatro polinômios.

$$
\begin{aligned}
& P_{1}^{p a r}(z)=a_{0}{ }^{-}+a_{2}{ }^{+} z^{2}+a_{4}{ }^{-} z^{4}+\ldots=\sum_{l=0, p a r}^{n} \mathbf{i}^{l} \cdot \min \left\{\mathbf{i}^{l} a_{l}{ }^{-}, \mathbf{i}^{l} a_{l}{ }^{+}\right\} z^{l} \\
& P_{2}^{\text {par }}(z)=a_{0}{ }^{+}+a_{2}{ }^{-} z^{2}+a_{4}{ }^{+} z^{4}+\ldots=\sum_{l=0, p a r}^{n} \mathbf{i}^{l} \cdot \max \left\{\mathbf{i}^{l} a_{l}^{-}, \mathbf{i}^{l} a_{l}{ }^{+}\right\} z^{l} \\
& P_{1}^{\text {impar }}(z)=a_{1}{ }^{-} z+a_{3}{ }^{+} z^{3}+a_{5}{ }^{-} z^{5}+\ldots=\sum_{l=0, \text { impar }}^{n} \mathbf{i}^{l-1} \cdot \min \left\{\mathbf{i}^{l-1} a_{l}{ }^{-}, \mathbf{i}^{l-1} a_{l}{ }^{+}\right\} z^{l} \\
& P_{2}^{\text {impar }}(z)=a_{1}{ }^{+} z+a_{3}{ }^{-} z^{3}+a_{5}{ }^{+} z^{5}+\ldots=\sum_{l=0, \text { impar }}^{n} \mathbf{i}^{l-1} \cdot \max \left\{\mathbf{i}^{l-1} a_{l}{ }^{-}, \mathbf{i}^{l-1} a_{l}{ }^{+}\right\} z^{l}
\end{aligned}
$$

Definição 4.1.1 Os polinômios de Kharitonov são:

$$
k_{m n}=P_{m}^{p a r}+P_{n}^{i m p a r}
$$

onde $m, n=1,2$.

É fácil ver que os polinômios de Kharitonov estão contidos no conjunto $K$. Observe que a escolha de $P_{m}^{\text {par }}$ e $P_{n}^{\text {impar }}$ é semelhante a de $P_{1}$ e $P_{2}$ de (3.13). $P_{m}^{\text {par }}$ e $P_{n}^{\text {impar }}$ são uma decomposição de $k_{m n}(\mathbf{i} w)$ em parte real e em parte imaginária. Então para todo $P \in K, \operatorname{Re} P(\mathbf{i} w) \in\left[P_{1}^{p a r}(\mathbf{i} w), P_{2}^{p a r}(\mathbf{i} w)\right]$ e $\operatorname{ImP}(\mathbf{i} w) \in\left[P_{1}^{\text {impar }}(\mathbf{i} w), P_{2}^{\text {impar }}(\mathbf{i} w)\right]$.

Definimos $M(w)=\{P(\mathbf{i} w) \mid P \in K\}$. 


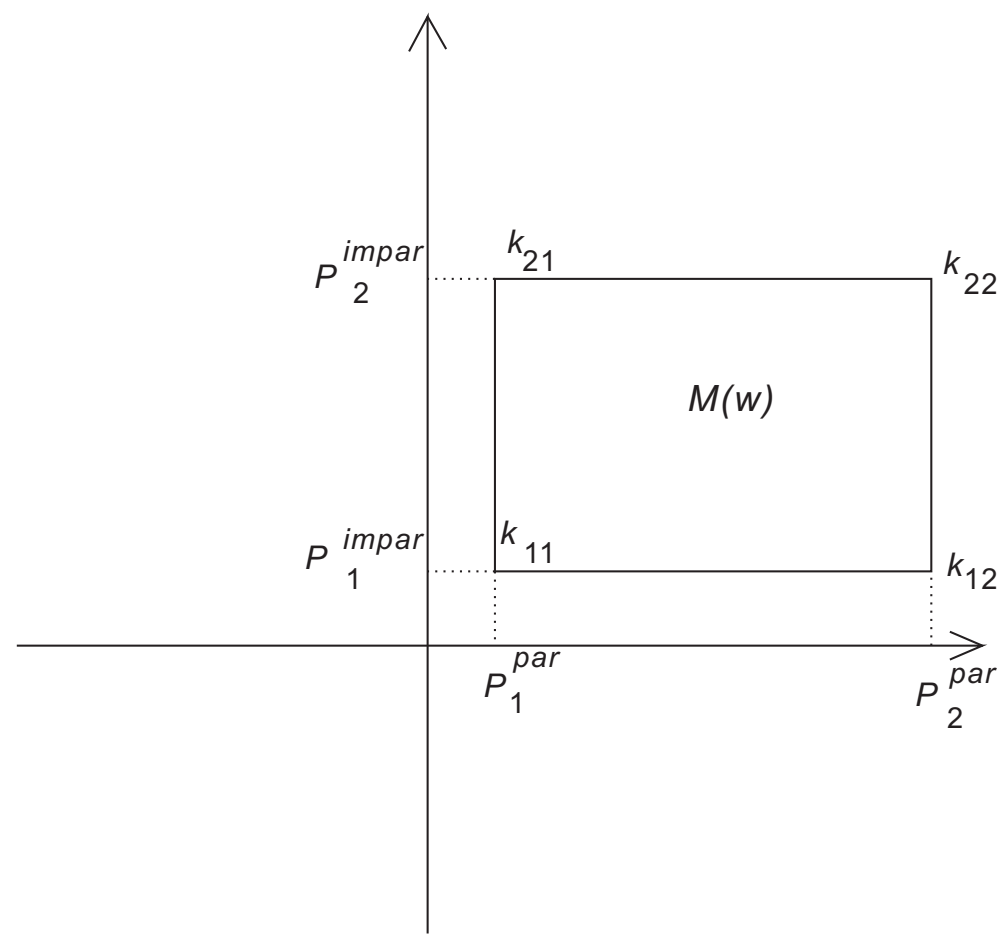

Figura 4.1: A imagem do retângulo $M(w)$

Os polinômios de Kharitonov calculados em $\mathbf{i} w, k_{11}(\mathbf{i} w), k_{12}(\mathbf{i} w), k_{21}(\mathbf{i} w)$ e $k_{22}(\mathbf{i} w)$, no plano complexo são as extremidades de um retângulo $M(w)$. Além disso, como a parte real e a parte imaginária das extremidades de $M(w)$ são os valores dos polinômios (4.2), (4.3), (4.4) e (4.5) em i $w$, o retângulo $M(w)$ mantém o seu formato ao longo da variação de $w$. Os lados continuam sendo paralelos aos eixos real e imaginário. Ver ilustração na figura 4.1. Este fato é importante para a demonstração do teorema de Kharitonov.

Lema 4.1.2 Se os polinômios de Kharitonov são de Hurwitz, então $0 \notin M(w)$ para todo $w \in \mathbb{R}^{+}$.

Demonstração Em particular, $k_{11}$ é polinômio de Hurwitz. Então $a_{0}{ }^{-}>0$ pela desigualdade (3.2). Se $w=0$, a parte imaginária dos polinômios de $M(0)$ é zero, mas $\operatorname{Re} M(0) \in\left[a_{0}{ }^{-}, a_{0}{ }^{+}\right]$. 


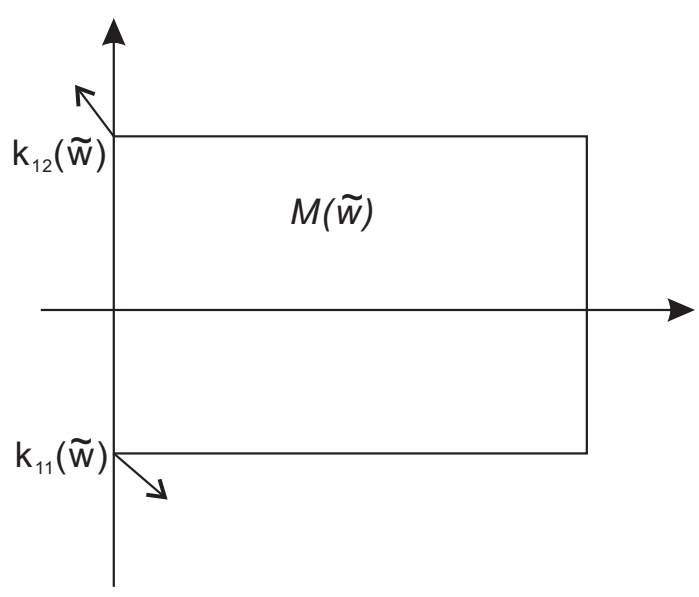

Figura 4.2: A contradição quando $0 \in M(w)$

Suponha, por absurdo, que existe $w>0$ tal que $0 \in M(w)$. Como $P(z) \in K$ são todos contínuos, $M(w)$ varia continuamente com $w$. No primeiro momento que $0 \in M(w), 0$ "toca"num dos lados (fronteira) de $M(w)$. Os vértices de $M(w)$ que são valores de polinômios de Hurwitz em i $w$ nunca se anulam, devido à proposição 3.1.3.

Desta forma, se $0 \in M(w)$, está numa das arestas, com exceção das esquinas, de $M(w)$. Suponha que o lado esquerdo de $M(\widetilde{w})$ tocou a origem, ou seja $P_{1}^{\text {par }}(\widetilde{w})=0$. Pela proposição 3.1.2, os polinômios de Hurwitz percorrem em sentido anti-horário em torno de 0 quando $w$ cresce. Então para $w>\widetilde{w}, \operatorname{Re} k_{11}(w)>0$, e ao mesmo tempo $\operatorname{Re} k_{12}(w)<0$. Mas isso não pode ocorrer, pois $\operatorname{Re} K_{11}(w)=\operatorname{Re} K_{12}(w)=P_{1}^{\text {par }}(w)$. Ver figura 4.2. Para os demais lados o argumento é análogo.

Teorema 4.1.3 Todos os $P(z) \in K$ são polinômios de Hurwitz se, e somente se, $k_{m n}$, onde $m, n=$ 1,2, são polinômios de Hurwitz.

Demonstração Seguiremos a demonstração do artigo [14]. Como $k_{m n} \in K$, se todos os polinômios no conjunto $K$ forem polinômios de Hurwitz, os $k_{m n}$ também são polinômios Hurwitz. 
Se os polinômios de Kharitonov forem de Hurwitz, como $P(\mathbf{i} w) \in M(w)$, pelo lema 4.1.2, $P(\mathbf{i} w) \neq$ 0. $M(w)$, por sua vez quando $w$ varia de 0 a $\infty$, em razão das extremidades serem os polinômios de Hurwitz e pela proposição 3.1.3, o ângulo percorrido por $M(w)$ é $\frac{n \pi}{2}$. Então $P(z)$ satisfaz às condições da proposição 3.1 .3 , logo $P(z)$ é polinômio de Hurwitz.

Nesta demonstração, usamos fortemente a propriedade geométrica. Há uma outra maneira de demonstração [4] na qual não é utilizada nenhuma propriedade geométrica, trabalhando-se num espaço de polinômios com coeficientes reais $\mathbb{R}^{n}$. Por exemplo, o espaço em que $\left(a_{0}, a_{1}, a_{2}, \ldots, a_{n-1}\right)$ representa um polinômio tal como

$$
P(z)=a_{0}+a_{1} z+a_{2} z^{2}+\ldots+a_{n-1} z^{n-1}
$$

Neste espaço, temos um paralelepípedo $K_{p}$ de dimensão $n$ que é delimitado pelo intervalo finito $a_{l} \in\left[a_{l}{ }^{-}, a_{l}{ }^{+}\right]$para todo $l=0,1,2,3, \ldots, n-1$ como dado em (4.1). A partir dos polinômios de Kharitonov, podemos definir um plano afim. De fato, $k_{11}, k_{12}, k_{21}, k_{22}$ estão num mesmo plano, pois

$$
k_{22}=-k_{11}+k_{12}+k_{21}
$$

Chamamos de plano de Kharitonov uma intersecção entre o paralelepípedo $K_{p}$ e o plano afim definido pelos polinômios de Kharitonov.

Todos os polinômios pertencentes a este plano definido pelos polinômios de Kharitonov são uma combinação convexa dos polinômios de Kharitonov. Esta pode ser uma outra definição para plano de Kharitonov.

Usamos dois fatos:

1) Se tiver um polinômio com uma raiz com parte real negativa no paralelepípedo $K_{p}$, temos um 
polinômio com uma raiz puramente imaginária no plano de Kharitonov. [4]

2) A combinação convexa de dois polinômios estáveis com os mesmos termos de grau par (ou ímpar) é um polinômio estável. [18]

A idéia da demonstração é supor por absurdo que existe um polinômio instável no $K_{p}$, então por 1) existe um polinômio instável em plano de Kharitonov. Isso é uma contradição, pois por 2) o plano de Kharitonov só tem polinômios de Hurwitz.

Não vamos demonstrar os fatos 1) e 2), pois este é um comentário sobre a prova alternativa para o teorema 4.1.3 de modo a explicitar o que ficou escondido na demonstração geométrica. O fato 1) usa fortemente a propriedade de continuidade dos zeros dos polinômios. O fato 2) usa a propriedade de que $K_{p}$ é conexo por caminhos.

Na demonstração geométrica os fatos 1) e 2) foram substituídos pelo argumento de que o retângulo se desloca no sentido anti-horário e pelo argumento do retângulo deslocar-se de maneira estável: $M(w)$ não "toca" 0 e percorre $n$ quadrantes. De fato, os polinômios de $K$ calculados em i $w$ são as combinações convexas de $k_{11}(\mathbf{i} w), k_{12}(\mathbf{i} w), k_{21}(\mathbf{i} w)$ e $k_{22}(\mathbf{i} w)$.

Observe que o teorema de Kharitonov pode ser estendido para qualquer conjunto $K_{1}$ contido em $K$ que contém os polinômios de Kharitonov. Se $K_{1}$ é de Hurwitz, $k_{m n}$ são de Hurwitz, $\operatorname{logo} K$ é de Hurwitz também e como $K_{1} \subset K, K_{1}$ é de Hurwitz. Assim temos a equivalência.

\subsubsection{Uma simplificação para os polinômios de grau menor que 6}

Pelo teorema de Kharitonov, sabemos que para qualquer $n$, basta checar apenas 4 polinômios para saber se todos polinômios de $K$ são de Hurwitz. Mas no exemplo 3.2.11, foi preciso analisar um único determinante, ou seja, um único polinômio para obter a estabilidade dos polinômios correspondetes à região hachurada da figura 3.11. O motivo é que as desigualdades dadas pelo teorema de Hurwitz 
para os polinômios de grau menor ficam redundantes. Vejamos um exemplo de polinômio de grau 4.

$$
P(z)=z^{4}+a_{1} z^{3}+a_{2} z^{2}+a_{3} z+a_{4}
$$

onde $a_{i} \in\left[a_{i}^{-}, a_{i}^{+}\right], a_{i}>0$, e $a_{i}^{-}>0$ para $i=1,2,3,4$.

Todos os coeficientes são positivos, satisfazendo a condição necessária para $P$ ser polinômio estável. Lembrando a desigualdade (3.69), obtemos a condição necessária e suficiente:

$$
a_{1} a_{2} a_{3}-a_{3}^{2}-a_{1}^{2} a_{4}>0
$$

Os polinômios de Kharitonov são

$$
\begin{aligned}
& k_{11}=z^{4}+a_{1}^{+} z^{3}+a_{2}^{+} z^{2}+a_{3}^{-} z+a_{4}^{-} \\
& k_{12}=z^{4}+a_{1}^{-} z^{3}+a_{2}^{+} z^{2}+a_{3}^{+} z+a_{4}^{-} \\
& k_{21}=z^{4}+a_{1}^{+} z^{3}+a_{2}^{-} z^{2}+a_{3}^{-} z+a_{4}^{+} \\
& k_{22}=z^{4}+a_{1}^{-} z^{3}+a_{2}^{-} z^{2}+a_{3}^{+} z+a_{4}^{+}
\end{aligned}
$$

Observe que se a condição de estabilidade do $k_{22}$ for satisfeita, a condição de estabilidade do $k_{12}$ também é satisfeita.

$$
\begin{aligned}
& a_{1}^{-} a_{2}^{-} a_{3}^{+}-a_{3}^{+2}-a_{1}^{-2} a_{4}^{+}>0 \\
& a_{1}^{-} a_{2}^{+} a_{3}^{+}-a_{3}^{+2}-a_{1}^{-2} a_{4}^{-}>0
\end{aligned}
$$

De fato, a condição de estabilidade de $k_{12}$ é redundante. Pela razão análoga, se a condição de estabilidade de $k_{21}$ for satisfeita, a condição do $k_{11}$ é satisfeita. De fato existe uma simplificação do 


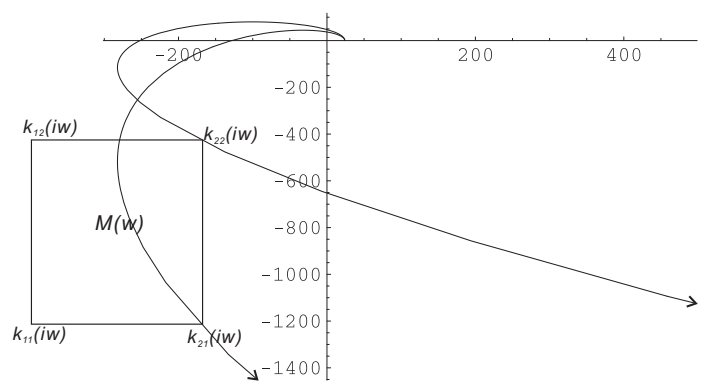

Figura 4.3: Polinômios de grau 4

teorema de Kharitonov no caso de grau menor que 6. Relembrando a proposição 3.1 .3 , o grau do polinômio é relacionado com o ângulo percorrido quando $w$ varia de zero até $\infty$. Novamente iremos usar o argumento geométrico para esta simplificação do teorema de Kharitonov.

Proposição 4.1.4 Para $n=3$, suponha que $a_{i}{ }^{-}>0$, então $K$ contém somente polinômios de Hurwitz se, e somente se $k_{21}$ é polinômio de Hurwitz.

Demonstração Se $K$ é de Hurwitz, é imediato que $k_{21}$ é de Hurwitz.

Se $k_{21}$ é de Hurwitz, observe que $M(w)$ varia do primeiro quadrante para o terceiro quadrante. Como $k_{21}$ corresponde ao vértice de lado direito inferior, se $k_{21}$ não se anular, o resto de conjunto também não se anula. Podemos ver isso na figura 3.11, todos os polinômios que correspondem a região hachurada em nenhum momento passam por 0 e o ângulo percorrido por $M(w)$ é $\frac{3 \pi}{2}$, satisfazendo a proposição 3.1.3. Todos os polinômios de $K$ são polinômios de Hurwitz.

Quando o grau do polinômio aumenta, aumenta também o ângulo total que $M(w)$ percorre. Então, além de verificar que $k_{12}(\mathbf{i} w) \neq 0$ para todo $w$, temos que verificar os outros polinômios de Kharitonov.

Proposição 4.1.5 Para $n=4$, suponha que $a_{i}{ }^{-}>0$, então $K$ só tem polinômios de Hurwitz se, $e$ 
somente se $k_{21}$ e $k_{22}$ são polinômios de Hurwitz.

Demonstração Se $K$ é estável, é imediato que $k_{21}$ e $k_{22}$ são polinômios de Hurwitz.

Observe que $M(w)$ percorre de primeiro quadrante para o quarto quadrante no sentido antihorário. Então para verificar que $M(w) \neq 0$ até o quarto quadrante, temos de checar $k_{21}$ como no caso de $\mathrm{n}=3$. Mesmo que $k_{21}(\mathbf{i} w)$ não toque zero até o quarto quadrante, temos a possibilidade de algum polinômio calculado em $\mathbf{i} w$ que corresponde o lado direito acima de $k_{21}(\mathbf{i} w)$ se anular. Mas se $k_{22}$ também for de Hurwitz, todos os polinômios de $K$ movimentam-se para o quarto quadrante sem se anularem. Ver figura 4.3. A partir disso o argumento é o mesmo da proposição 4.1 .4 para concluir que $K$ é um conjunto de polinômios estáveis.

Proposição 4.1.6 Para $n=5$, suponha que $a_{i}{ }^{-}>0$. Então $K$ é um conjunto de polinômios de Hurwitz se, e somente se, $k_{21}, k_{22}$ e $k_{12}$ são polinômios de Hurwitz.

Demonstração Se $K$ é um conjunto de polinômios de Hurwitz, é imediato que $k_{21}$ e $k_{22}$ são de Hurwitz.

$\mathrm{O}$ fato de $k_{21}, k_{22}$ serem estáveis nos garante que até o quarto quadrante não existe nenhum polinômio de $K$ passando por zero. Ao voltar para o primeiro quadrante, temos uma possibilidade do lado superior de $M(w)$ tocar 0 . Isso não ocorre se $k_{12}$ for polinômio de Hurwitz, garantindo que $k_{11}$ não toque zero. O resto da demonstração é análogo a outras proposições anteriores.

A partir de $n=6$, precisamos checar todos os 4 polinômios de Kharitonov.

\subsection{Aplicação do teorema de Kharitonov}

Vimos até agora o critério de Routh-Hurwitz e o teorema de Kharitonov que são condições para caracterizar a estabilidade dos sistemas do tipo (2.2), (2.5) e (2.6). Vamos aplicar esta teoria de 
estabilidade para um problema de controle da retro-alimentação.

Primeiro apresentamos a forma matricial do teorema de Kharitonov [3] para o seguinte sistema. Seja $A$ uma matriz $n \times n$.

$$
\dot{x}=A x
$$

onde $x=x(t) \in \mathbb{R}^{n}$

A forma matricial do teorema de Kharitonov de sistema de matriz (4.17) é a seguinte. Consideramos uma família de matrizes

$$
\mathcal{A}=\left\{\left[\begin{array}{cc}
0_{(n-1) \times 1} & I_{n-1} \\
-\mathcal{B}_{0} & \ldots-\mathcal{B}_{n-4}-\mathcal{B}_{n-3}-\mathcal{B}_{n-2}-\mathcal{B}_{n-1}
\end{array}\right]: \mathcal{B}_{i}^{-} \leq \mathcal{B}_{i} \leq \mathcal{B}_{i}^{+}, i=0, \ldots, n-1\right\}
$$

e as quatro matrizes

$$
\begin{aligned}
& A_{1}=\left[\begin{array}{cc}
0_{(n-1) \times 1} & I_{n-1} \\
\ldots-\mathcal{B}_{n-4}^{-}-\mathcal{B}_{n-3}^{-} & -\mathcal{B}_{n-2}^{+}-\mathcal{B}_{n-1}^{+}
\end{array}\right] \\
& A_{2}=\left[\begin{array}{cc}
0_{(n-1) \times 1} & I_{n-1} \\
\ldots-\mathcal{B}_{n-4}^{+}-\mathcal{B}_{n-3}^{-} & -\mathcal{B}_{n-2}^{-}-\mathcal{B}_{n-1}^{+}
\end{array}\right] \\
& A_{3}=\left[\begin{array}{cc}
0_{(n-1) \times 1} & I_{n-1} \\
\ldots-\mathcal{B}_{n-4}^{+}-\mathcal{B}_{n-3}^{+} & -\mathcal{B}_{n-2}^{-}-\mathcal{B}_{n-1}^{-}
\end{array}\right] \\
& A_{4}=\left[\begin{array}{cc}
0_{(n-1) \times 1} & I_{n-1} \\
\ldots-\mathcal{B}_{n-4}^{-}-\mathcal{B}_{n-3}^{+} & -\mathcal{B}_{n-2}^{+}-\mathcal{B}_{n-1}^{-}
\end{array}\right] .
\end{aligned}
$$

Primeiro, definimos a matriz estável.

Definição 4.2.1 As matrizes são estáveis se a parte real de todas as raízes do polinômio carac- 
terístico são negativas.

Proposição 4.2.2 Todas as matrizes da família $\mathcal{A}$ são estáveis se, e somente se, as matrizes $A_{1}, A_{2}, A_{3}, A_{4}$ são estáveis.

Demonstração Primeiro explicitamos o sistema 4.17.

$$
\begin{aligned}
& {\left[\begin{array}{c}
\dot{x} \\
\dot{x_{1}} \\
\dot{x_{2}} \\
\dot{x_{3}} \\
\vdots \\
x_{n-1}
\end{array}\right]=\left[\begin{array}{cccccc}
0 & 1 & 0 & \ldots & 0 & 0 \\
0 & 0 & 1 & \ldots & 0 & 0 \\
\vdots & \vdots & \vdots & \ddots & \vdots & \vdots \\
0 & 0 & 0 & \ldots & 1 & 0 \\
0 & 0 & 0 & \ldots & 0 & 1 \\
-\mathcal{B}_{0} & -\mathcal{B}_{1} & -\mathcal{B}_{2} & \ldots & -\mathcal{B}_{n-2} & -\mathcal{B}_{n-1}
\end{array}\right]\left[\begin{array}{c}
x \\
x_{1} \\
x_{2} \\
x_{3} \\
\vdots \\
x_{n-1}
\end{array}\right]} \\
& {\left[\begin{array}{c}
\dot{x} \\
\dot{x_{1}} \\
\dot{x_{2}} \\
\dot{x_{3}} \\
\vdots \\
x_{n-1}
\end{array}\right]=\left[\begin{array}{c}
x_{1} \\
x_{2} \\
x_{3} \\
x_{4} \\
\vdots \\
-\mathcal{B}_{0} x-\mathcal{B}_{1} x_{1}-\mathcal{B}_{2} x_{2}-\cdots-\mathcal{B}_{n-2} x_{n-2}-\mathcal{B}_{n-1} x_{n-1}
\end{array}\right]}
\end{aligned}
$$

que é equivalente à

$$
\mathcal{B}_{0} x+\mathcal{B}_{1} \dot{x}+\mathcal{B}_{2} \ddot{x}+\cdots+\mathcal{B}_{n-2} x^{(n-2)}+\mathcal{B}_{n-1} x^{(n-1)}+x^{(n)}=0
$$


cujo polinômio característico é

$$
\chi(s)=\mathcal{B}_{0}+\mathcal{B}_{1} s+\mathcal{B}_{2} s^{2}+\cdots+\mathcal{B}_{n-2} s^{n-2}+\mathcal{B}_{n-1} s^{n-1}+s^{n} .
$$

Observe que o conjunto dos polinômios característicos das matrizes da família $\mathcal{A}$ coincide o conjunto $K$ do teorema de Kharitonov, ver 4.1. Os polinômios característicos das matrizes $A_{1}, A_{2}, A_{3}$ e $A_{4}$ são os polinômios de Kharitonov 4.1.1.

Observe que valem as mesmas simplificações para $n$ menor que 6 , basta analisar a matiz correspondente para as proposições 4.1.4, 4.1.5 e 4.1.6 (neste caso dimensão de matriz coincide com grau de polinômio característico).

Apresentamos um problema de controle robusto da retro-alimentação. Sejam as matrizes $A_{n \times n}$, $B_{n \times m}, C_{l \times n}$. Consideramos o seguinte sistema dinâmico.

$$
\begin{gathered}
\dot{x}=A x+B u, x(0)=x_{o}, \\
y=C x \\
u=H y
\end{gathered}
$$

onde $x=x(t) \in \mathbb{R}^{n}, A \in \mathcal{A}$ é propriedade natural (por exemplo, o valor que gravidade, força de Coriolis e centrífuga) do mecanismo.

Em ponto de vista de mecanismo, do valor da entrada $x$ e do controlador $C$ temos o valor de saída $y=y(t) \in \mathbb{R}^{l}$. $y$ é parâmetro da entrada no mecanismo. O mecanismo exerce processo $H$ e dá 
valor de saída $u$. Este $u$ é detectado pelo sensor ( $u$ está num espaço diferente de entrada $x$ ), o sensor interpreta o valor e devolve o valor $B u$ que está no mesmo espaço do valor de entrada. Este valor de entrada é somado com o valor de estado anterior. Assim temos um sistema fechado de controle de retro-alimentação

$$
\dot{x}=(A+B H C) x
$$

O nosso problema é determinar $C$ para que (4.27) seja estável para qualquer $A \in \mathcal{A}$. Observe que o processo $H$ que o mecanismo exerce e o valor que sensor interpreta e devolve $B$ é constante. O lugar onde podemos escolher o valor é $C$. Em alguns casos de problemas de controle, $B, H$ e $C$ podem aparecer como uma única matriz $B H C$ como controlador. Claro que para entender a estabilidade, podemos aplicar o teorema de Kharitonov.

Consideramos que a matriz $B, n \times m$, tenha a forma

$$
B=\left[\begin{array}{c}
0_{n-1 \times m} \\
b
\end{array}\right]
$$

onde $b$ tem dimensões $1 \times m$. É natural $B$ ter esta forma, pois os termos de controle só afetam a n-ésima linha do sistema.

Corolário 4.2.3 Fixemos uma matriz $H_{m \times l}$ e consideremos o conjunto de matrizes

$$
\tilde{\mathcal{A}} \doteq\{A+B H C: A \in \mathcal{A}\}
$$

Então todas as matrizes de $\tilde{\mathcal{A}}$ são estáveis se, e somente se, $\tilde{A}_{i} \doteq A_{i}+B H C, i=1,2,3,4$, são estáveis. 


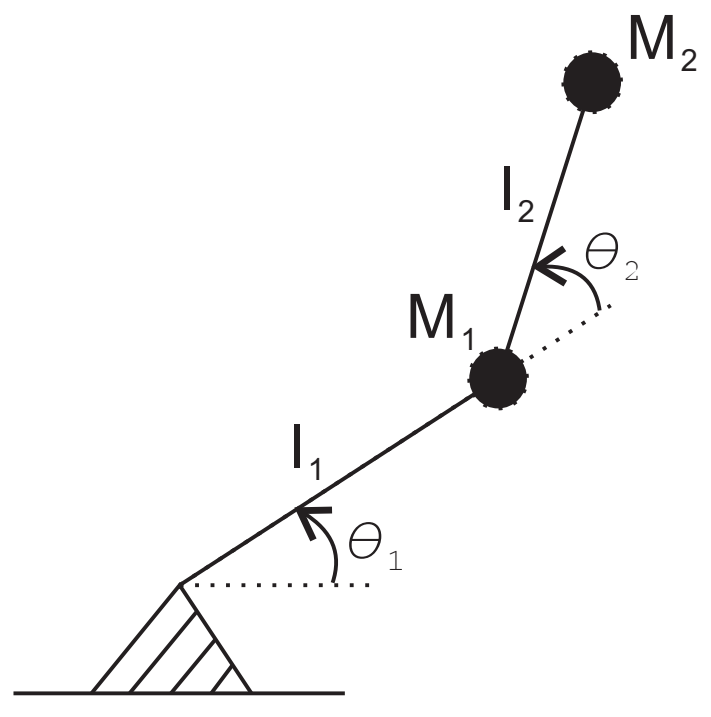

Figura 4.4: manipulador

Demonstração Todas as matrizes de $\tilde{\mathcal{A}}$ são da forma

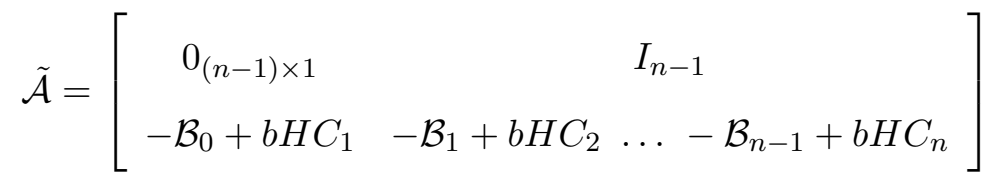

onde $C_{i}$ é $i$-ésima coluna de $C$. Definimos $\delta_{i} \doteq \mathcal{B}_{i}-b H C_{i}, \delta_{i}^{-} \doteq \mathcal{B}_{i}^{-}+b H C_{i}, \delta_{i}^{+} \doteq \mathcal{B}_{i}^{+}+b H C_{i}$. Apliquemos a proposição 4.2 .2 para

$$
\tilde{\mathcal{A}}=\left\{\left[\begin{array}{cc}
0_{(n-1) \times 1} & I_{n-1} \\
-\delta_{0} & \ldots-\delta_{n-4}-\delta_{n-3}-\delta_{n-2}-\delta_{n-1}
\end{array}\right]: \delta_{i}^{-} \leq \delta_{i} \leq \delta_{i}^{+}, i=0, \ldots, n-1\right\}
$$

As quatro matrizes dadas pela proposição são exatamente $\tilde{A}_{i} \doteq A_{i}+B H C, i=1,2,3,4$.

Vejamos um exemplo. Consideremos a dinâmica do robô manipulador [12]: 


$$
M(\theta) \ddot{\theta}+C(\theta, \dot{\theta})+G(\theta)=F
$$

onde $\theta(t)$ é vetor de articulação em função do tempo, $M$ é a matriz de inércia, simétrica, positiva definida, que depende da configuração, $C$ é o vetor de força de Coriolis e centrífuga, que é quadrática em $\dot{\theta}, G$ é o vetor de força gravitational e $F$ é o vetor de força aplicada na articulação. O controle PID (controle com termo proporcional, integral e diferencial) é dado por

$$
F=-K_{I} \int_{0}^{t}\left[\theta(\tau)-\theta_{d}\right] d \tau-K_{P}\left[\theta-\theta_{d}\right]-K_{D}\left[\dot{\theta}-\dot{\theta_{d}}\right]+G(\theta)
$$

onde $\theta_{d}$ é constante e $K_{D}, K_{P}, K_{I}$ são matriz de controlador simétrico que dá a entrada.

Definimos $\epsilon \doteq \int_{0}^{l}\left[\theta-\theta_{d}\right] d \tau$ e linearizando a expressão combinada de dinâmica de robô e controlador, no ponto de equilíbrio $(\epsilon, \theta, \dot{\theta})=\left(0, \theta_{d}, 0\right)$ temos

$$
M\left(\theta_{d}\right) \epsilon^{(3)}+K_{D} \ddot{\epsilon}+K_{P} \dot{\epsilon}+K_{I} \epsilon=0 .
$$

O problema é achar $K_{D}, K_{P}, K_{I}$ tal que a equação (4.37) seja estável para cada ponto $\theta_{d}$. Supomos que o movimento do robô é contínuo e o vetor de articulação $\theta$ está num conjunto compacto $\Theta$. Então existem matrizes definidas positivas $M^{+}$e $M^{-}$tais que $M^{-} \leq M(\theta) \leq M^{+}$, para $\forall \theta \in \Theta$. Vamos encontrar as condições em que o sistema seja estável.

A função característica da equação (4.37) é

$$
\chi(s)=\operatorname{det}\left[M\left(\theta_{d}\right) s^{3}+K_{D} s^{2}+K_{P} s+K_{I}\right]
$$

Seja $\lambda$ a raiz da função (4.38) para $\theta_{d} \in \Theta$ fixo. Seja $L$ o conjunto de todas as raízes de equação 
(4.38). Então temos um autovetor $\nu_{\lambda}$ associada a $\lambda$ e vale

$$
\left[M\left(\theta_{d}\right) \lambda^{3}+K_{D} \lambda^{2}+K_{P} \lambda+K_{I}\right] \nu_{\lambda}=0
$$

multiplicando no lado esquerdo a função conjugada transposta de $\nu_{\lambda}, \nu_{\lambda}^{*}$, temos um polinômio em $\lambda$ com coeficientes reais

$$
a_{3} \lambda^{3}+a_{2} \lambda^{2}+a_{1} \lambda+a_{0}=0
$$

onde $a_{3}=\nu_{\lambda}^{*} M\left(\theta_{d}\right) \nu_{\lambda}, a_{2}=\nu_{\lambda}^{*} K_{D} \nu_{\lambda}, a_{1}=\nu_{\lambda}^{*} K_{P} \nu_{\lambda}, a_{0}=\nu_{\lambda}^{*} K_{I} \nu_{\lambda}$.

$M$ tem máximo $M^{+}$e mínimo $M^{-} . K_{D}, K_{P}, K_{I}$ são controladores cujos valores podemos escolher. Fixamos os valores de $K_{D}, K_{P}, K_{I}$ ou podemos considerar que estão dentro de algum intervalo definido pelo mecanismo. Neste caso, para simplificar, escolhemos um valor fixo. Então temos intervalos que definem a familia de polinômios de 4.40

$$
a_{i} \in\left[a_{i}^{-}, a_{i}^{+}\right],
$$

para $i=0,1,2,3$, onde

$$
\begin{gathered}
a_{3}^{-}=\min _{\lambda \in L}\left\{\nu_{\lambda}{ }^{*} M \nu_{\lambda}: M^{-} \leq M \leq M^{+}\right\} \\
a_{3}^{+}=\max _{\lambda \in L}\left\{\nu_{\lambda}{ }^{*} M \nu_{\lambda}: M^{-} \leq M \leq M^{+}\right\} \\
a_{2}^{-}=\min _{\lambda \in L}\left\{\nu_{\lambda}{ }^{*} K_{D} \nu_{\lambda}\right\} \\
a_{2}^{+}=\max _{\lambda \in L}\left\{\nu_{\lambda}{ }^{*} K_{D} \nu_{\lambda}\right\}
\end{gathered}
$$




$$
\begin{array}{r}
a_{1}^{-}=\min _{\lambda \in L}\left\{\nu_{\lambda}{ }^{*} K_{P} \nu_{\lambda}\right\} \\
a_{1}^{+}=\max _{\lambda \in L}\left\{\nu_{\lambda}{ }^{*} K_{P} \nu_{\lambda}\right\}
\end{array}
$$

$$
\begin{array}{r}
a_{0}^{-}=\min _{\lambda \in L}\left\{\nu_{\lambda}{ }^{*} K_{I} \nu_{\lambda}\right\} \\
a_{0}^{+}=\max _{\lambda \in L}\left\{\nu_{\lambda}{ }^{*} K_{I} \nu_{\lambda}\right\}
\end{array}
$$

Usemos a simplificação do teorema de Kharitonov para $n=3$. Analisemos

$$
\chi(\lambda)=a_{3}^{+} \lambda^{3}+a_{2}^{-} \lambda^{2}+a_{1}^{-} \lambda+a_{0}^{+} .
$$

Se supusermos que $M, K_{D}, K_{P}, K_{I}$ são matrizes positivas definidas, a condição necessária para polinômio estável $\left(a_{i}^{-}>0, i=1,2, a_{i}^{+}>0, i=0,3\right)$ é satisfeita. Usando o critério de Hurwitz, a condição suficiente é

$$
a_{1}^{-} a_{2}^{-}-a_{3}^{+} a_{0}^{+}>0
$$

Descreveremos um procedimento para escolher o valor de $K_{D}, K_{I}, K_{P}$ a partir do limitante superior da matriz de inércia $M^{+}$. Vamos analisar a equação característica

$$
\chi(s)=\operatorname{det}\left[M^{+}\left(\theta_{d}\right) s^{3}+K_{D} s^{2}+K_{P} s+K_{I}\right]
$$

Como as raízes da equação característica (4.52) não variam, mesmo se multiplicarmos por $M^{+\left(-\frac{1}{2}\right)}=$ ${\sqrt{M^{+}}}^{(-1)}$ pelo lado direito e pelo lado esquerdo, temos:

$$
\chi(s)=\operatorname{det}\left[I s^{3}+\widetilde{K_{D}} s^{2}+\widetilde{K_{P}} s+\widetilde{K_{I}}\right],
$$


onde

$$
\begin{aligned}
\widetilde{K_{D}} & =M^{+\left(-\frac{1}{2}\right)} K_{D} M^{+\left(-\frac{1}{2}\right)}, \\
\widetilde{K_{P}} & =M^{+\left(-\frac{1}{2}\right)} K_{P} M^{+\left(-\frac{1}{2}\right)}, \\
\widetilde{K_{I}} & =M^{+\left(-\frac{1}{2}\right)} K_{I} M^{+\left(-\frac{1}{2}\right)} .
\end{aligned}
$$

Simplificamos a equação, escolhendo $\widetilde{K_{D}}, \widetilde{K_{P}}$ e $\widetilde{K_{I}}$ como

$$
\widetilde{K_{D}}=k_{d} I, \widetilde{K_{P}}=k_{p} I, \quad \widetilde{K_{I}}=k_{i} I
$$

Os valores de controle são:

$$
K_{D}=k_{d} M^{+}, \quad K_{P}=k_{p} M^{+}, \quad K_{I}=k_{i} M^{+}
$$

Da desigualdade (4.51), o polinômio (4.52) é estável se

$$
k_{i}<k_{d} k_{p}
$$

Escolhemos $k_{i}, k_{d}$ e $k_{p}$ de tal maneira que a desigualdade (4.59) seja satisfeita. Então a equação (4.52) e (4.53) são estáveis. Seja $\lambda$ a raiz de (4.53), $\nu_{\lambda}$ o autovetor associado.

Observe que a equação (4.51) continua valendo para os valores $a_{3}$ tal que $a_{3}^{+} \geq a_{3}>0$

$$
a_{1}^{-} a_{2}^{-}-a_{3} a_{0}^{+}>0
$$

Isto significa que se o polinômio característico com $a_{3}^{+}$for estável, o polinômio característico para $a_{3}$ também é estável. $a_{1}^{-}, a_{2}^{-}$e $a_{0}^{+}$são valores fixos definidos a partir de $k_{i}, k_{d}$ e $k_{p}$ que são valores 
escolhidos tais que satisfaçam a desigualdade (4.59).

Como $\nu_{\lambda}{ }^{*} M \nu_{\lambda} \leq \nu_{\lambda}{ }^{*} M^{+} \nu_{\lambda}$ para qualquer $M$ tal que $M \leq M^{+}$a função característica (4.52) for estável,

$$
\chi(s)=\operatorname{det}\left[M s^{3}+K_{D} s^{2}+K_{P} s+K_{I}\right]
$$

também é estável para $M \leq M^{+}$.

Então para obter $k_{i}, k_{d}$ e $k_{p}$ que garantem a estabilidade de função característica $\chi(s)=\operatorname{det}\left[M s^{3}+\right.$ $\left.K_{D} s^{2}+K_{p} s+K_{l}\right], M \leq M^{+}$, basta encontrar os $k_{i}, k_{d}$ e $k_{p}$ que satisfaçam a condição de estabilidade da função característica (4.53), que equivale à função característica com $M^{+}$. 


\section{Capítulo 5}

\section{Conclusões}

\subsection{Considerações finais}

Neste trabalho, apresentamos vários critérios que caracterizam os polinômios estáveis, isto é aqueles com todas suas raízes com parte real negativa, também chamado de polinômio de Hurwitz. Trabalhamos com polinômios $P$ de grau $n$, com todos os coeficientes reais. Mais precisamente, focalizamos os polinômios com todos os coeficientes diferentes de zero e de mesmo sinal, pois estes são os polinômios que satisfazem a condição necessária para a estabilidade.

Para encontrar uma condição suficiente, analisamos a relação entre o sinal da parte real, o número das raízes com parte real negativa e o comportamento de $P(\mathbf{i} w)$, focando quantos quadrantes percorre $P(\mathbf{i} w)$ e em que sentido o faz.

Usando esta relação, obtivemos o seguinte critério suficiente com cunho geométrico: Para um polinômio ser de Hurwitz, $P(\mathbf{i} w) \neq 0$ deve percorrer exatamente $n$ quadrantes no sentido anti-horário.

Desta equivalência, resulta que o problema de caracterizar o polinômio estável se torna um problema de análise das raízes dos polinômios $P_{1}\left(w^{2}\right)$ (parte real) e $P_{2}\left(w^{2}\right)$ (parte imaginária) do polinômio $P(\mathbf{i} w)$ de (3.13) . O fato de $P(\mathbf{i} w)$ percorrer $n$ quadrantes é equivalente ao fato dos polinômios 
$P_{1}\left(w^{2}\right)$ e $P_{2}\left(w^{2}\right)$ terem $\left[\frac{n}{2}\right]$ e $\left[\frac{n-1}{2}\right]$ raízes reais, que estão intercaladas entre si.

A equivalência que relaciona os coeficientes de polinômio $P$ e as propriedades das raízes de $P_{1}\left(w^{2}\right)$ e $P_{2}\left(w^{2}\right)$ de um polinômio estável é o critério de Routh 3.2.7. Ele usa o seguinte esquema para definir os números $c_{p, q}$ :

$$
\begin{array}{c|ccc} 
& c_{1,0}=a_{0}, & c_{2,0}=a_{2}, & c_{3,0}=a_{4}, \ldots \\
c_{1,1}=a_{1}, & c_{2,1}=a_{3}, & c_{3,1}=a_{5}, \ldots \\
r_{2}=\frac{a_{0}}{a_{1}} & c_{1,2}=c_{2,0}-r_{2} c_{2,1}, & c_{2,2}=c_{3,0}-r_{2} c_{3,1}, & c_{3,2}=c_{4,0}-r_{2} c_{4,1}, \ldots \\
r_{3}=\frac{c_{1,1}}{c_{1,2}} & c_{1,3}=c_{2,1}-r_{3} c_{2,2}, & c_{2,3}=c_{3,1}-r_{3} c_{3,2}, & c_{3,3}=c_{4,1}-r_{3} c_{4,2}, \ldots \\
\hline r_{q}=\frac{c_{1, q-2}}{c_{1, q-1}} & \multicolumn{3}{c}{c_{p, q}=c_{p+1, q-2}-r_{q} c_{p+1, q-1}, \ldots} \\
\end{array}
$$

onde $p=1,2,3, \ldots$ e $q=2,3,4, \ldots$.

De cada linha $c_{p, q}, p=1,2,3, \cdots$, podemos construir de uma maneira conveniente uma seqüência de polinômios, para os quais $c_{1, q}, q=1,2,3, \cdots$ são os coeficientes do termo de maior grau. Isto é, são os principais fatores que decidem os sinais dos polinômios. A partir dos primeiros dois polinômios desta seqüência, podemos criar os polinômios que equivalem a $P_{1}\left(w^{2}\right)$ e $P_{2}\left(w^{2}\right)$ de (3.13). Como as raízes estão fortemente relacionadas com o número de trocas de sinal, fazendo uma análise do número de trocas de sinal desta seqüência podemos obter as condições que satisfazem o critério da estabilidade dadas pelas raízes dos $P_{1}\left(w^{2}\right)$ e $P_{2}\left(w^{2}\right)$. Um polinômio é de Hurwitz se, e somente se, satisfaz a condição necessária e $c_{1, q}>0$ para todo $q=1,2,3, \cdots$. No capítulo 3 , demonstramos o teorema de Hurwitz que estabelece o seguinte: condição de estabilidade de polinômio é que todos os determinantes dos menores $H_{i}(\underline{3.22})$ da matriz (3.17) sejam positivos. Este fato é equivalente a $c_{1, q}>0, q=1,2,3, \cdots$.

Vimos alguns exemplos (3.73) que ilustram o fato de que com um polinômio estável podemos 
encontrar outros polinômios estáveis, devido ao fato das condições necessárias e suficientes de Routh e Hurwitz serem dadas pelas desigualdades (3.54) e (3.67). Foi apresentada uma extensão deste exemplo para qualquer grau $n$ que é o teorema de Kharitonov. Este teorema trata de uma família $K$ (4.1) de polinômios cujos coeficientes são delimitados por intervalos compactos. Os polinômios $P(\mathbf{i} w)$ do conjunto $K$ formam um retângulo no plano complexo e os polinômios de Kharitonov (4.1.1) são as esquinas deste retângulo. O comportamento dos polinômios neste retângulo é determinado pelo comportamento dos polinômios de Kharitonov. Assim, todos os polinômios pertencentes a $K$ são estáveis, se e somente se os polinômios de Kharitonov são estáveis.

Para verificar a estabilidade de uma familia de polinômios de grau menor que 6 não precisamos analisar todos os polinômios de Kharitonov, pois a estabilidade de um certo polinômio já resulta estabilidade de outro polinômio de Kharitonov. Assim, mostramos uma simplificação do teorema de Kharitonov para grau menor que 6. Para os polinômios de grau igual a 3, só precisamos checar a estabilidade de $k_{21}$. Para os de grau 4, basta checar a estabilidade de $k_{21}$ e $k_{22}$. Para os de grau 5 , temos que checar a estabilidade de $k_{21}, k_{22}$ e $k_{12}$. Para os de grau 6, devemos checar a estabilidade dos quatro polinômios de Kharitonov.

Finalizamos com um problema de estabilidade de um sistema de equações diferenciais. O sistema é estável se o seu polinômio característico é estável. Usando o teorema de Kharitonov, podemos verificar a estabilidade de uma família de sistemas. Aplicamos esta idéia num problema de controle por retro-alimentação. Obtivemos o conjunto $K$ a partir do sistema, encontrando os polinômios característicos do mesmo. Aplicamos o teorema de Kharitonov para encontrar as desigualdades que envolvem os parâmetros do sistema. Se os parâmetros do problema satisfizerem as desigualdades, o sistema resulta estável. 


\subsection{Sugestões para estudo adicional}

Uma linha de pesquisa seria generalizar o teorema de Kharitonov nas direções esboçadas a seguir.

Nesta dissertação, trabalhamos apenas com polinômios com coeficientes reais. É de interesse matemático estender os resultados para polinômios com coeficientes complexos. O próprio Kharitonov 22] estabeleceu esta generalização em 1978, sendo que uma prova mais simples foi apresentada por Bose e Chi [15]. Podemos, ainda, encontrar uma outra demonstração em [14]. Nesta generalização, o número de polinômios a serem testados para estabelecer a estabilidade é 8 .

Uma outra direção da pesquisa consiste em deixar variável o grau dos polinômios. Até agora, fixamos o grau dos polinômios como sendo $n$ e usamos fortemente este fato na prova dos resultados. É possível garantir a estabilidade, mesmo que os coeficientes se anulem. Isto é, permitir que $0 \leq$ $a_{i}^{-} \leq a_{i} \leq a_{i}^{+}, \mathrm{i}=0,1,2,3, \ldots, \mathrm{n}$, para um polinômio $P(z)=\sum_{i=o}^{n} a_{i} z^{i}$. Os polinômios desta familia são estáveis se, e somente se, os polinômios de Kharitonov são de Hurwitz. A prova está em [6].

Podemos trabalhar com sistemas com atraso no tempo. Por exemplo,

$$
\dot{y}=\alpha y(t), y(t)+\beta y(t-\tau)=0
$$

A solução é

$$
y(t)=e^{\alpha_{0} t}
$$

satisfazendo $P\left(\alpha_{0}\right)=1+\beta e^{-\alpha_{0} \tau}=0$. O problema consiste em analisar as raízes destes $P$. Se todas as raízes de $P$ têm parte real negativa, temos estabilidade. Chamamos de quase-polinômios àqueles da forma $P$. Como a função para analisar as raízes não é mais um polinômio, temos algumas dificuldades. Como não temos um grau fixo como nos polinômios, $P$ pode ter infinitas raízes. Neste contexto, podemos criar um resultado semelhante ao teorema de Kharitonov. O estudo detalhado 
está em [16]. 


\section{Referências Bibliográficas}

[1] B. D. O Anderson, E. I. Jury, , and M. Mansour, On robust hurwitz polynomials, IEEE Transactions on automatic control AC-32 (1987), no. 10, 909-913. 43

[2] Yury S. Barkovsky, Lectures on the routh-hurwitz problem, arXiv:0802.1805v1(http://arxiv.org/abs/0802.1805v1), 2008. 17, 20, 31

[3] Dennis S. Berstein and Wassim M. Haddad, Robust controller synthesis using kharitonov's theorem, IEEE Transactions on automatic control 37 (1992), no. 1, 129-132. 52

[4] Hervé Chapellat and S.P Bhattacharyya, An alternative proof of khatitonov's theorem, IEEE Transactions on automatic control 34 (1989), no. 4, 448-450. 43, 47, 48

[5] W. Hahn, Stability of motion, Springer-Verlag, New York, 1967. 9

[6] R. Hernández and S. Dormido, Kharitonov's theorem extension to interval polynomials which can drop in degree: a nyquist approach, IEEE Transactions on automatic control 41 (1996), no. 7, 1009-1012. 66

[7] Olga Holtz, Hermite-biehler, routh-hurwitz, and total positivity, Linear Algebra and its Applications 372 (2003), 105-110. 20

[8] A. Hurwitz, Über die bedingungen unter welchen eine gleichung nur wurzeln mit negativen reelen teilen besitzt, Mathematische Annalen 46 (1895), 273-280. 2

[9] V.L. Kharitonov, Asymptotic stability of an equilibrium position of a family of systems of linear differential equations, Differential Equations 14 (1979), 1483-1485. 3

[10] A. G. J. MacFarlane, The development of frequency-response methods in automatic control, IEEE T. Automat. Contr. AC-24 (1979), 250-265.

[11] J. C. Maxwell, On governors, Proc. R. Soc. 16 (1868), 270-283. 2

[12] Tesfay Meressi, Degang Chen, and Brad Paden, Application of kharitonov's theorem to mechanical systems, IEEE Transactions on automatic control 38 (1993), no. 3, 488-491. 56 
[13] Antony. N. Michel, Stability: The common thread in the evolution of feedback control, IEEE Control Systems 16 (1996), no. 3, 50-60. 2

[14] R. J. Minnichelli, J. J. Anagnost, and C.A. Desoer, An elementary proof of khatitonov's stability theorem with extensions, IEEE Transactions on automatic control 34 (1989), no. 9, 995-998. 43, 46, 66

[15] N.K.Bose and Y.Q.Shi, A simple general proof of kharitonov's generalized stability criterion, IEEE Trans. Circuits Syst. CAS-34 (1987), 1233-1237. 66

[16] Vadim Olshevsky and M. Lev Sakahnovich, A generalized kharitonov theorem for quasipolynomials and entire functions occurring in systems with multiple and distributed delays, Advanced Signal Processing Algorithms, Architectures, and Implementations XV, ed. F.T Luk, SPIE publications (2005), 325-336. 20, 67

[17] E. J. Routh, A treatise on the stability of a given state of motion, Macmillan, London (1877). 2

[18] S.Bialas and J. Garloff, Convex combinations of stable polynomials, J. Franklin Inst. 319 (1985), 373-377. 48

[19] Jorge Sotomayor, Luis Fernando Mello, and Denis de Carvalho Braga, Bifurcation analysis of the watt governor systems, Comp. Appl. Math 26 (2007), no. 1, 19-44. 3

[20] Jorge Sotomayor Tello, Lições de equações diferenciais ordinárias, Projeto Euclides, 1979. 6]

[21] F.R. Gantmacher translated by K.A. Hirsch, The theory of matrices, vol 1,2, Chelsea Publishing Co., New York, 1959. 17, 20, 31

[22] V.L.Kharironov, On a generalization of a stability criterion, Izvestiia Akademii nauk Kazakhskoi SSR, Seria fiziko-mathematicheskaia 1 (1978), 53-57. 66 


\section{Índice Remissivo}

Critério de Hurwitz, 39

Critério de Routh, 21

Estabilidade de Liapunov, 5

Polinômio de Hurwitz, 8

Polinômio estável, 8

Teorema de Kharitonov, 46 